\title{
Regular solutions of DAE hybrid systems and regularization techniques *
}

\author{
Peter Kunkel ${ }^{\dagger} \quad$ Volker Mehrmann ${ }^{\ddagger}$
}

18.04.17

\begin{abstract}
The solvability and regularity of hybrid differential-algebraic systems (DAEs) is studied, and classical stability estimates are extended to hybrid DAE systems. Different reasons for non-regularity are discussed and appropriate regularization techniques are presented. This includes a generalization of Filippov regularization in the case of so-called chattering. The results are illustrated by several numerical examples.
\end{abstract}

Keywords. Differential-algebraic equation, hybrid system, switched system, index reduction, existence and uniqueness of solutions, Filippov regularization, strangeness index, chattering.

AMS(MOS) subject classification. 34K34, 34K28, 65L80, 65L05, 34K32

\section{Introduction}

Differential-algebraic equations (DAEs) are widely used in the modeling process of complex dynamical systems, see, e. g., [3, 9]. In many applications the mathematical system equations switch between different models. One then speaks of switched or hybrid systems, see, e. g., [4, 6, 22]. Classical application areas of switched systems are robot manipulators [17], mechatronics [16], mechanical systems with dry friction [10, 20], or automatic gear-boxes [15]. Other applications include electronic circuits where different device models are used for different frequency ranges, or switching elements like diodes are used, see [22], process control in chemical engineering [1], and in control systems $[24,26]$, where the value of a control switches between different operation modes. See also $[12,18]$ for further examples. For an overview of modeling, analysis, simulation and control of hybrid systems, see, e. g., [4, 23, 24, 25].

In this paper we study the solvability of general nonlinear hybrid systems of differential-algebraic equations and extend the work of $[14,15,24,25]$. We characterize the existence of regular solutions and when no regular solutions exist. We also present stability estimates for general hybrid systems of DAEs and derive regularization techniques that allow the numerical integration.

A hybrid system of DAEs on a (nontrivial) closed interval $\mathbb{I}=[\underline{t}, \bar{t}] \subseteq \mathbb{R}$ consists of model equations describing the different modes of the hybrid system, switching conditions describing when a mode loses its validity, and transition functions describing the initiation of a new mode. Hence, a hybrid system of DAEs with $J$ modes is described as follows. The model equations are given by nonlinear DAEs of the form

$$
\begin{aligned}
& F^{j}\left(t, x^{j}, \dot{x}^{j}\right)=0, \quad j=1, \ldots, J, \\
& F^{j}: \mathbb{I} \times \mathbb{D}_{x}^{j} \times \mathbb{D}_{\dot{x}}^{j} \rightarrow \mathbb{R}^{n^{j}},
\end{aligned}
$$

\footnotetext{
*Supported through the Research-in-Pairs Program at Mathematisches Forschungsinstitut Oberwolfach.

${ }^{\dagger}$ Mathematisches Institut, Universität Leipzig, Augustusplatz 10, D-04109 Leipzig, Fed. Rep. Germany, kunkel@math.uni-leipzig.de. Supported by Deutsche Forschungsgemeinschaft under grant no. KU964/7-1.

${ }^{\ddagger}$ Institut für Mathematik, MA 4-5, Technische Universität Berlin, D-10623 Berlin, Fed. Rep. Germany, mehrmann@math.tu-berlin.de. Supported by European Research Council through the Advanced Grant MODSIMCONMP.
} 
where $\mathbb{D}_{x}^{j}, \mathbb{D}_{x}^{j} \subseteq \mathbb{R}^{n^{j}}$ denote the open domains of definition of the variables $x^{j}$ and $\dot{x}^{j}$ in the arguments of $F^{j}$.

We assume each of these mode systems to be regular in the sense that they satisfy Hypothesis 4.2 of [19], see Section 2 below. In particular, for each mode we have a set $\mathbb{L}_{\mu^{j}}^{j}$ describing the possible consistent initial conditions for this mode.

The switching conditions have the form

$$
\begin{aligned}
& S_{l}^{j}\left(t, x^{j}\right)=0, \quad l=1, \ldots, L^{j}, \quad j=1, \ldots, J, \\
& S_{l}^{j}: \mathbb{I} \times \mathbb{D}_{x}^{j} \rightarrow \mathbb{R},
\end{aligned}
$$

together with the so-called feasible regions

$$
\mathbb{F}^{j}=\left\{\left(t, x^{j}\right) \in \mathbb{I} \times \mathbb{D}_{x}^{j} \mid S_{l}^{j}\left(t, x^{j}\right) \geq 0, l=1, \ldots, L^{j}\right\}
$$

in which the solution is required to lie if the system is in the $j$ th mode.

The transition functions have the form

$$
T_{l}^{k j}: \mathbb{I} \times \mathbb{D}_{x}^{j} \rightarrow \mathbb{L}_{\mu^{k}}^{k}, \quad l=1, \ldots, L^{j}, \quad j=1, \ldots, J,
$$

where $k=\sigma^{j}(l)$, with $\sigma^{j}:\left\{1, \ldots, L^{j}\right\} \rightarrow\{1, \ldots, J\}$, is the new mode when mode $j$ was terminated due to a sign change in the switching function $S_{l}^{j}$.

For our analysis we will assume that all functions are sufficiently smooth, i. e., sufficiently often continuously differentiable. In particular, we assume that all occurring derivatives exist.

The paper is organized as follows. In Section 2 we recall the basics of differential-algebraic equations and in Section 3 we discuss the regularity of hybrid DAE systems. The extension of classical stability estimates to hybrid DAE systems is treated in Section 4. Possible non-regular behavior and regularization techniques for hybrid DAEs, including a generalization of the Filippov regularization, are presented in Section 5 and some numerical examples are presented in Section 6 . We conclude with a summary in Section 7 .

\section{Preliminaries}

In order to introduce the concept of regularity for solutions of hybrid DAE systems, the DAEs of the single modes must be regular. To recall the regularity of general DAEs, let $F$ describe a nonlinear DAE

$$
\begin{aligned}
& F(t, x, \dot{x})=0, \\
& F: \mathbb{I} \times \mathbb{D}_{x} \times \mathbb{D}_{\dot{x}}, \quad \mathbb{D}_{x}, \mathbb{D}_{\dot{x}} \subseteq \mathbb{R}^{n} \text { open },
\end{aligned}
$$

as it is given in each mode of (1). Following an idea of Campbell, see, e. g., [7], we consider so-called derivative array equations

$$
\begin{aligned}
& F_{\mu}(t, x, y)=0, \quad y=\left(\dot{x}, \ldots, x^{(\mu+1)}\right), \\
& F_{\mu}: \mathbb{I} \times \mathbb{D}_{x} \times \mathbb{D}_{y} \rightarrow \mathbb{R}^{(\mu+1) n}, \quad \mathbb{D}_{x} \subseteq \mathbb{R}^{n}, \mathbb{D}_{y} \subseteq \mathbb{R}^{(\mu+1) n} \text { open, }
\end{aligned}
$$

defined by stacking $F$ and its first $\mu$ derivatives of $F$ on top of each other, thus

$$
F_{\mu}=\left[\begin{array}{c}
F \\
\frac{d}{d t} F \\
\vdots \\
\left(\frac{d}{d t}\right)^{\mu} F
\end{array}\right] .
$$

According to [19], regularity of the DAE (5) can be guaranteed by requiring the following hypothesis, in which by $F_{\mu ; z}$ we denote the Jacobian of $F_{\mu}$ with respect to the variable $z$. 
Hypothesis 1 Consider a DAE of the form (5). There exist integers $\mu$, a, and $d$ such that the set

$$
\mathbb{L}_{\mu}=F_{\mu}^{-1}(\{0\})
$$

associated with $F$ is nonempty and such that for every point $\left(t_{0}, x_{0}, y_{0}\right) \in \mathbb{L}_{\mu}$, there exists a (sufficiently small) neighborhood $\mathbb{U}$ in which the following properties hold:

1. We have $\operatorname{rank} F_{\mu ; y}=(\mu+1) n-a$ on $\mathbb{L}_{\mu} \cap \mathbb{U}$ such that there exists a smooth matrix function $Z_{2}$ of size $(\mu+1) n \times a$ and pointwise maximal rank, satisfying $Z_{2}^{T} F_{\mu ; y}=0$ on $\mathbb{L}_{\mu} \cap \mathbb{U}$.

2. We have rank $Z_{2}^{T} F_{\mu ; x}=a$ on $\mathbb{U}$ such that there exists a smooth matrix function $T_{2}$ of size $n \times d, d=n-a$, and pointwise maximal rank, satisfying $Z_{2}^{T} F_{\mu ; x} T_{2}=0$ on $\mathbb{U}$.

3. We have $\operatorname{rank} F_{\dot{x}} T_{2}=d$ on $\mathbb{U}$ such that there exists a smooth matrix function $Z_{1}$ of size $n \times d$ and pointwise maximal rank, satisfying $\operatorname{rank} Z_{1}^{T} F_{\dot{x}} T_{2}=d$ on $\mathbb{U}$.

If a DAE system satisfies Hypothesis 1, then the smallest $\mu$ for which this is the case is called the strangeness index of $F$ and systems with $\mu=0$ are called strangeness-free.

We will utilize Hypothesis 1 in the following form. Given a solution $x^{*}: \mathbb{I} \rightarrow \mathbb{R}^{n}$ of the derivative array system $F_{\mu}=0$, in the sense that there exists a continuous path $\left(t, x^{*}(t), \mathcal{P}(t)\right) \in \mathbb{L}_{\mu}$ for all $t \in \mathbb{I}$ with $\dot{x}^{*}=\mathcal{P}\left[\begin{array}{ll}I_{n} & 0\end{array}\right]^{T}$, Hypothesis 1 guarantees the existence of (smooth) functions $\hat{F}_{1}, \hat{F}_{2}$ defining a so-called reduced $D A E$

$$
\begin{array}{ll}
\hat{F}_{1}(t, x, \dot{x})=0, & (d \text { differential equations }) \\
\hat{F}_{2}(t, x)=0, & (a \text { algebraic equations })
\end{array}
$$

with $x^{*}$ being a solution of (6). Moreover, the reduced DAE satisfies Hypothesis 1 with $\mu=0$ and, therefore, possesses solutions for sufficiently small perturbations of the initial value $x^{*}\left(t_{0}\right)$ within the set $\left\{x_{0} \in \mathbb{R}^{n} \mid \hat{F}_{2}\left(t_{0}, x_{0}\right)=0\right\}$.

A (smooth) transformation of the variable $x$ according to

$$
x=Q\left[\begin{array}{l}
x_{1} \\
x_{2}
\end{array}\right],
$$

where $Q: \mathbb{I} \rightarrow \mathbb{R}^{n \times n}$ is pointwise orthogonal, then yields a decoupled DAE of the form

$$
\begin{array}{ll}
\dot{x}_{1}=\mathcal{L}\left(t, x_{1}\right), & (d \text { differential equations }) \\
x_{2}=\mathcal{R}\left(t, x_{1}\right), & (a \text { algebraic equations })
\end{array}
$$

with appropriate (smooth) functions $\mathcal{L}, \mathcal{R}$. For details, see [19].

Since for given $\hat{t}$, every $\left(t_{0}, x_{0}, y_{0}\right) \in \mathbb{L}_{\mu}$ in a neighborhood $\mathbb{U}$ of $\left(\hat{t}, x^{*}(\hat{t}), \mathcal{P}(\hat{t})\right)$ fixes a solution of the reduced DAE, we have a flow $\Phi_{t, t_{0}}: \mathbb{U} \rightarrow \mathbb{I} \times \mathbb{R}^{n}$ for all $t$ in a sufficiently small neighborhood of $t_{0}$. The flow maps $\left(t_{0}, x_{0}, y_{0}\right)$ to $(t, x(t))$, where $x(t)$ is the value of the corresponding solution at $t$. Note that the existence of a (global) solution on the whole of $\mathbb{I}$ guarantees the existence of $\Phi_{t, t_{0}}$ for all $t \in \mathbb{I}$ provided $\mathbb{U}$ is chosen sufficiently small.

\section{Regular solutions of hybrid systems of DAEs}

To study the regularity of hybrid systems of DAEs, we assume that every mode in (1) is regular in the sense that $F^{j}$ satisfies Hypothesis 1 with some characteristic values $\mu^{j}, a^{j}, d^{j}$ and a set $\mathbb{L}_{\mu^{j}}^{j}$ of possible consistent initial conditions, as it was introduced in Hypothesis 1 . Using then the decoupled index reduced formulation (8) for each mode,

$$
\dot{x}_{1}^{j}=\mathcal{L}^{j}\left(t, x_{1}^{j}\right), \quad x_{2}^{j}=\mathcal{R}^{j}\left(t, x_{1}^{j}\right), \quad j=1, \ldots, J,
$$

one obtains transformed switching conditions (2) which have the form

$$
\mathcal{S}_{l}^{j}\left(t, x_{1}^{j}\right)=0, \quad l=1, \ldots, L^{j}, \quad j=1, \ldots, J
$$


after eliminating the variables $x_{2}$ with the help of the algebraic equations in (9). Accordingly, the transition functions can be transformed to

$$
\mathcal{T}_{l}^{k j}\left(t, x_{1}^{j}\right)=\left(t, x_{1}^{k}\right), \quad l=1, \ldots, L^{j}, \quad j=1, \ldots, J, \quad k \in\{1, \ldots, J\} .
$$

Remark 2 If a switching condition is given in the more general form

$$
S_{l}^{j}\left(t, x^{j}, \dot{x}^{j}\right)=0
$$

then it should reduce to the form

$$
\mathcal{S}_{l}^{j}\left(t, x_{1}^{j}, x_{2}^{j}, \dot{x}_{1}^{j}\right)=0
$$

before the elimination of $x_{2}^{j}$. In particular, there should be no argument $\dot{x}_{2}^{j}$, since the presence of $\dot{x}_{2}^{j}$ would result in the need of a further differentiation of $F^{j}$. This requirement corresponds to the possibility in the ODE case to eliminate differentiated arguments with the help of the differential equation.

In order to define a regular solution of a hybrid system on a given fixed interval $\left[t_{0}, T\right]$, we must define regular solutions of the single modes (which is already obtained by requiring Hypothesis 1 for each mode) as well as a regular switching behavior. Thus, we first discuss the regularity of a piece of the overall solution that corresponds to a specific mode. In the following, we call these pieces of the solution regular arcs.

Definition 3 Consider a hybrid DAE (1) in the interval $\mathbb{I}=[\underline{t}, \bar{t}]$ and let $j \in\{0, \ldots, J\}$. A function $x \in C^{1}\left(\mathbb{I}, \mathbb{R}^{n^{j}}\right)$ is called a regular arc (of mode $j$ ) if

1. there exists $\underline{z}=(\underline{x}, \underline{y})$ such that $(\underline{t}, \underline{z}) \in \mathbb{L}_{\mu^{j}}^{j}$ and $(t, x(t))=\Phi_{t, \underline{t}}^{j}\left(\underline{t}, \underline{z}^{j}\right)$ for all $t \in \mathbb{I}$;

2. the initial value is feasible according to $(\underline{t}, \underline{x}) \in \mathbb{F}^{j}$ and there exists an $\varepsilon^{j}>0$ and a neighborhood $\mathbb{U}$ of $(\underline{t}, \underline{z})$ such that

$$
\Phi_{t, t_{0}}^{j}\left(t_{0}, z_{0}\right) \in \stackrel{\circ}{F^{j}}
$$

for all $\left(t_{0}, z_{0}\right) \in \mathbb{U}$ and all $t \in\left(t_{0}, t_{0}+\varepsilon^{j}\right]$, where $\stackrel{\circ}{F}^{j}$ denotes the interior of the set $\mathbb{F}^{j}$;

3. there exists $\hat{l} \in\left\{1, \ldots, L^{j}\right\}$ such that

(a) the end point $\bar{t}$ is a regular solution of the one-dimensional problem $\left(S_{\hat{l}}^{j} \circ \Phi_{t, \underline{t}}^{j}\right)(\underline{t}, \underline{z})=0$ in the sense that $\left.\frac{d}{d t}\left(S_{\hat{l}}^{j} \circ \Phi_{t, \underline{t}}^{j}\right)(\underline{t}, \underline{z})\right|_{t=\bar{t}} \neq 0$,

(b) strict feasibility holds according to $\left(S_{\hat{l}}^{j} \circ \Phi_{t, \underline{t}}^{j}\right)(\underline{t}, \underline{z})>0$ for all $t \in(\underline{t}, \bar{t})$,

(c) strict feasibility holds with respect to the other switching functions up to the end point according to $\left(S_{l}^{j} \circ \Phi_{t, \underline{t}}^{j}\right)(\underline{t}, \underline{z})>0$ for all $t \in(\underline{t}, \bar{t}]$ and all $l \in\left\{1, \ldots, L^{j}\right\}$ with $l \neq \hat{l}$.

Remark 4 The conditions in Definition 3 can be interpreted as follows. Condition 1. just states that $x$ is solution of the DAE of mode $j$ belonging to the (consistent) initial condition given by $x(\underline{t})=\underline{x}$. Condition 2. requires that the initial state is feasible and the corresponding solution stays strictly feasible according to

$$
\left(S_{l}^{j} \circ \Phi_{t, t_{0}}^{j}\right)\left(t_{0}, z_{0}\right)>0, \quad j=1, \ldots, J
$$

for sufficiently small perturbations of the initial condition at least for some small time interval whose length can be bounded away from zero. Condition 3. requires that the end point is a regular solution of a unique switching function along the trajectory and that no other switching condition was satisfied before.

To simplify the notation in the following definition of a regular solution of a hybrid system, we assume that every mode contains the termination condition $t-\bar{t}=0$ as a switching condition. 
Definition 5 Consider a hybrid DAE (1) in the interval $\mathbb{I}=[\underline{t}, \bar{t}]$. Given a collection of $N \in \mathbb{N}$ switching points

$$
\mathbf{t}=\left(t_{0}, t_{1}, \cdots, t_{N}\right), \quad \underline{t}=t_{0}<t_{1}<\cdots<t_{N}=\bar{t}
$$

and a collection of modes

$$
\mathbf{j}=\left(j_{0}, j_{1}, \cdots, j_{N-1}\right), \quad j_{i} \in\{1, \ldots, J\} \text { for } i=0, \ldots, N-1,
$$

a finite collection

$$
\mathbf{x}=\left(x_{0}^{j_{0}}, x_{1}^{j_{1}}, \ldots, x_{N-1}^{j_{N-1}}\right)
$$

of functions $x^{j_{i}} \in C^{1}\left(\left[t_{i}, t_{i+1}\right], \mathbb{R}^{n^{j_{i}}}\right)$ is called a regular solution of the hybrid system (1) if $x^{j_{i}}$ is a regular arc of mode $j_{i}$ for every $i=0, \ldots, N-1$, and the following condition holds:

Let

$$
\mathbf{z}=\left(z_{0}^{j_{0}}, z_{1}^{j_{1}}, \ldots, z_{N-1}^{j_{N-1}}\right)
$$

be the corresponding initial values and let

$$
\mathbf{l}=\left(l_{0}, l_{1}, \ldots, l_{N-1}\right)
$$

be the uniquely defined activated switching functions according to the definition of a regular arc. Then the transitions satisfy

$$
j_{i+1}=\sigma^{j_{i}}\left(l_{i}\right)
$$

and

$$
\left(t_{i+1}, z_{i+1}^{j_{i+1}}\right)=\left(T_{l_{i}}^{j_{i+1}, j_{i}} \circ \Phi_{t_{i+1}, t_{i}}^{j_{i}}\right)\left(t_{i}, z_{i}^{j_{i}}\right), \quad i=0, \ldots, N-2 .
$$

Remark 6 A regular solution according to Definition 5 has the following properties. Since we require a finite switching structure given by $\mathbf{t}$ and $\mathbf{j}$, there is a common $\varepsilon=\min _{j \in\{0, \ldots, J\}} \varepsilon^{j}>0$ for all arcs of a regular solution. By assumption, the activated switching condition in mode $j_{N-1}$ of the last interval is given by $t-\bar{t}=0$ which leads to the termination of the integration process for the hybrid system.

The notion of a regular solution is justified by the following result.

Theorem $\mathbf{7}$ Consider a hybrid system of the form (1) and let $\mathbf{x}$ with corresponding $\mathbf{t}$ and $\mathbf{j}$ be a regular solution of the given hybrid system. Then (1) possesses a regular solution for every initial condition $x^{j_{0}}\left(t_{0}\right)=\tilde{x}_{0}$ with $\left(t_{0}, \tilde{x}_{0}^{j_{0}}, \tilde{y}_{0}^{j_{0}}\right) \in \mathbb{L}_{\mu^{j_{0}}}^{j_{0}}$ from a sufficiently small neighborhood of $\left(t_{0}, x_{0}^{j_{0}}, y_{0}^{j_{0}}\right)=\left(t_{0}, z_{0}^{j_{0}}\right)$ and the final value $\tilde{x}^{j_{N-1}}\left(t_{N}\right)$ depends smoothly on $\tilde{x}_{0}$.

Proof. Since there exists a solution of the hybrid system, there is a flow $\Phi_{t, \tilde{t}}^{j_{i}}\left(\tilde{t}, \tilde{z}_{i}^{j_{i}}\right)$ for all consistent $\left(\tilde{t}, \tilde{z}_{i}^{j_{i}}\right)$ in a neighborhood of $\left(t_{i}, z_{i}^{j_{i}}\right)$ and all $t$ in a neighborhood of $\left[t_{i}, t_{i+1}\right]$.

By assumption, the switching point $t_{i+1}$ is a regular solution of

$$
S_{l_{i}}^{j_{i}}\left(\Phi_{t, t_{i}}^{j_{i}}\left(t_{i}, z_{i}^{j_{i}}\right)\right)=0
$$

Hence, by the implicit function theorem, see, e. g., [21], there exists a (smooth) function $R_{l_{i}}^{j_{i}}$ with

$$
S_{l_{i}}^{j_{i}}\left(\Phi_{\hat{t}, \tilde{t}}^{j_{i}}\left(\tilde{t}, \tilde{z}_{i}^{j_{i}}\right)\right) \equiv 0 \text { for } \hat{t}=R_{l_{i}}^{j_{i}}\left(\tilde{t}, \tilde{z}_{i}^{j_{i}}\right) .
$$

Since the sign conditions hold for all switching functions $S_{l}^{j_{l}}$, the so obtained $\hat{t}$ remains the first switching point that occurs for all consistent $\left(\tilde{t}, \tilde{z}_{i}^{j_{i}}\right)$ in a neighborhood of $\left(t_{i}, z_{i}^{j_{i}}\right)$. As a consequence, at $\hat{t}$ the system changes from mode $j_{i}$ to mode $j_{i+1}$ and $\Phi_{\hat{t}, \tilde{t}}^{j_{i}}\left(\tilde{t}, \tilde{z}_{i}^{j_{i}}\right)$ depends smoothly on consistent initial values $\left(\tilde{t}, \tilde{z}_{i}^{j_{i}}\right)$ in an appropriate neighborhood of $\left(t_{i}, z_{i}^{j_{i}}\right)$. Hence,

$$
\left(\tilde{t}, \tilde{z}_{i+1}^{j_{i+1}}\right)=T_{l_{i}}^{j_{i+1}, j_{i}}\left(\Phi_{\hat{t}, \tilde{t}}^{j_{i}}\left(\tilde{t}, \tilde{z}_{i}^{j_{i}}\right)\right) \text { with } \hat{t}=R_{l_{i}}^{j_{i}}\left(\tilde{t}, \tilde{z}_{i}^{j_{i}}\right)
$$


depends smoothly on $\left(\tilde{t}, \tilde{z}_{i}^{j_{i}}\right)$ in an appropriate neighborhood of $\left(t_{i}, z_{i}^{j_{i}}\right)$ and is consistent for the mode $j_{i+1}$ due to the required properties of $T_{l_{i}}^{j_{i+1}, j_{i}}$.

In this way we have shown that small changes in the initial conditions of a mode do not change the switching that is activated and only yield small changes in the initial condition for the next mode.

Putting all modes together, we have shown that there exists a neighborhood of $\left(t_{0}, z_{0}^{j_{0}}\right)$ such that for all consistent $\left(t_{0}, \tilde{z}_{0}^{j_{0}}\right)$ from this neighborhood the corresponding initial value problem for (1) possesses a solution with the same collection of modes and the same activated switching functions given both by the sequence $\mathbf{j}$. The switching points $\tilde{t}_{i}$ and initial values $\tilde{z}_{i}^{j_{i}}$ depend smoothly on the initial value $\left(t_{0}, \tilde{z}_{0}^{j_{0}}\right)$. In particular, the final value $\tilde{x}^{j_{N-1}}\left(t_{N}\right)$ is a smooth function of the initial value $\left(t_{0}, \tilde{z}_{0}^{j_{0}}\right)$.

Remark 8 It follows from Theorem 7 that we have

$$
\left(t_{N}, x^{j_{N-1}}\right)=\Psi\left(t_{0}, z_{0}^{j_{0}}\right)
$$

with a smooth function

$$
\Psi=\Phi_{t_{N}, t_{N-1}}^{j_{N-1}} \circ \bigodot_{i=0}^{N-2}\left(T_{l_{i}}^{j_{i+1}, j_{i}} \circ \Phi_{t_{i+1}, t_{i}}^{j_{i}}\right)
$$

where

$$
t_{i+1}=R_{l_{i}}^{j_{i}}\left(t_{i}, z_{i}^{j_{i}}\right)=\left(R_{l_{i}}^{j_{i}} \circ \bigcirc_{m=0}^{i-1}\left(T_{l_{m}}^{j_{m+1}, j_{m}} \circ \Phi_{t_{m+1}, t_{m}}^{j_{m}}\right)\right)\left(t_{0}, z_{0}^{j_{0}}\right) .
$$

Remark 9 If suitable spaces for a Banach space formulation of an initial value problem for hybrid DAE systems are needed, as for example in the treatment of optimal control problems for hybrid systems, then it is necessary to transform the switching structure to a fixed grid. This can for example be done via a linear transformation

$$
\theta^{i}:\left[t_{i}, t_{i+1}\right] \rightarrow[i, i+1], \quad \theta^{i}(t)=\frac{t-t_{i}}{t_{i+1}-t_{i}}+k .
$$

To summarize, we have introduced the concept of regular solutions for hybrid systems of DAEs and we have shown that such a solution stays regular in a small neighborhood with the same switching structure and that we obtain a flow of the system for the whole interval $\mathbb{I}$ under consideration. In the next section we derive a stability estimate for such regular solutions.

\section{$4 \quad$ Stability estimates}

For ordinary differential equations

$$
\dot{x}=f(t, x)
$$

there is a well-known stability estimate which states that (under suitable assumptions) the solution depends Lipschitz-continuously on perturbations in the initial value and in the evaluation of the right hand side of the ODE, see, e. g., [13, Theorem I.10.3].

Theorem 10 Let $x \in C^{1}\left(\left[t_{0}, t_{1}\right], \mathbb{R}^{n}\right)$ be a solution of (10) in a real interval $\left[t_{0}, t_{1}\right]$ and let $\tilde{x} \in$ $C^{1}\left(\left[t_{0}, t_{1}\right], \mathbb{R}^{n}\right)$ satisfy

(a) $\quad\left\|\tilde{x}\left(t_{0}\right)-x\left(t_{0}\right)\right\| \leq \delta$

(b) $\quad\|\dot{\tilde{x}}(t)-f(t, \tilde{x}(t))\| \leq \beta$ for all $t \in\left[t_{0}, t_{1}\right]$,

(c) $\|f(t, \tilde{x}(t))-f(t, x(t))\| \leq L\|\tilde{x}(t)-x(t)\|$ for all $t \in\left[t_{0}, t_{1}\right]$,

with some constant $L>0$. Then

$$
\|\tilde{x}(t)-x(t)\| \leq e^{L\left(t-t_{0}\right)} \delta+\frac{1}{L}\left(e^{L\left(t-t_{0}\right)}-1\right) \beta \text { for all } t \in\left[t_{0}, t_{1}\right] .
$$


In this section we derive a corresponding stability estimate for hybrid systems of DAEs, which includes possible perturbations in all parts of the involved computations thus generalizing Theorem 7 .

We first consider hybrid ODEs and consider a single mode given by (10) together with a regular $\operatorname{arc} x \in C^{1}\left(\left[t_{j}, t_{j+1}\right], \mathbb{R}^{n}\right)$. This arc is governed by a unique switching condition denoted here by

$$
S(t, x)=0 .
$$

In particular, we assume that $f$ and $S$ are defined on a compact neighborhood of

$$
\left\{(t, x(t)) \mid t \in\left[t_{j}, t_{j+1}\right]\right\} \subseteq \mathbb{R} \times \mathbb{R}^{n}
$$

and that

$$
\text { (a) } S\left(t_{j+1}, x\left(t_{j+1}\right)\right)=0, \quad \frac{d}{d t} S\left(t_{j+1}, x\left(t_{j+1}\right)\right) \neq 0 \text {, }
$$

(b) $\quad S(t, x(t))>0$ for all $t \in\left(t_{j}, t_{j+1}\right)$.

In this way, the terminal point $t_{j+1}$ is fixed by being the first zero larger than $t_{j}$ of the function

$$
u(t)=S(t, x(t)) .
$$

Suppose that $\tilde{x} \in C^{1}\left(\left[\tilde{t}_{j}, \tilde{t}_{j+1}\right], \mathbb{R}^{n}\right)$ is a perturbed solution which satisfies

(a) $\quad\left\|\tilde{x}\left(\tilde{t}_{j}\right)-x\left(t_{j}\right)\right\| \leq \delta$

(b) $\quad\left|\tilde{t}_{j}-t_{j}\right| \leq \tau$,

(c) $\quad\|\dot{\tilde{x}}(t)-f(t, \tilde{x}(t))\| \leq \beta$ for all $t \in\left[t_{j}, t_{j+1}\right]$,

(d) $\quad\left|S\left(\tilde{t}_{j+1}, \tilde{x}\left(\tilde{t}_{j+1}\right)\right)\right| \leq \sigma$,

for sufficiently small $\delta, \tau, \beta, \sigma>0$. Our goal is to estimate $\left\|\tilde{x}\left(\tilde{t}_{j+1}\right)-x\left(t_{j+1}\right)\right\|$ and $\left|\tilde{t}_{j+1}-t_{j+1}\right|$. Note, however, that the correct terminal point $\tilde{t}_{j+1}$ under perturbations may not be the first zero of the switching function, since under perturbations we cannot guarantee the feasibility of the initial value.

For the correct choice of the terminal point, we first observe that if $\tau$ is sufficiently small, then the solution $x$ can be extended to $\tilde{t}_{j}$ even if $\tilde{t}_{j}$ is not contained in $\left[t_{j}, t_{j+1}\right]$. Since $f$ is bounded on its compact domain of definition, say by a constant $M$, then it holds that

$$
\begin{gathered}
\left\|x\left(\tilde{t}_{j}\right)-x\left(t_{j}\right)\right\|=\left\|\left.x\left(t_{j}+s\left(\tilde{t}_{j}-t_{j}\right)\right)\right|_{0} ^{1}\right\|=\left\|\int_{0}^{1} \dot{x}\left(t_{j}+s\left(\tilde{t}_{j}-t_{j}\right)\right)\left(\tilde{t}_{j}-t_{j}\right) d s\right\| \\
\leq \max _{s \in[0,1]}\left\|f\left(t_{j}+s\left(\tilde{t}_{j}-t_{j}\right), x\left(t_{j}+s\left(\tilde{t}_{j}-t_{j}\right)\right)\right)\right\|\left|\tilde{t}_{j}-t_{j}\right| d s \leq M \tau .
\end{gathered}
$$

It follows from this inequality and (12a) that

$$
\left\|\tilde{x}\left(\tilde{t}_{j}\right)-\tilde{x}\left(t_{j}\right)\right\|=\left\|\tilde{x}\left(\tilde{t}_{j}\right)-x\left(t_{j}\right)\right\|+\left\|x\left(\tilde{t}_{j}\right)-x\left(t_{j}\right)\right\| \leq \delta+M \tau,
$$

and Theorem 10 applied to the interval $\left[t_{j}, t_{j+1}\right]$ implies that

$$
\|\tilde{x}(t)-x(t)\| \leq e^{L\left(t-t_{j}\right)}(\delta+M \tau)+\frac{1}{L}\left(e^{L\left(t-t_{j}\right)}-1\right) \beta,
$$

as long as both functions $x$ and $\tilde{x}$ are defined and satisfy

$$
\|f(t, \tilde{x}(t))-f(t, x(t))\| \leq L\|\tilde{x}(t)-x(t)\|
$$

with some constant $L>0$.

Having studied the influence of a perturbation in the initial state and during the integration of the system, we now must discuss the influence of the perturbations on the accepted switching point $\tilde{t}_{j+1}$.

Since we only detect switching points by the change of sign of the switching function along the computed solution, it is natural to assume that both $x$ and $\tilde{x}$ are still defined in a neighborhood 
of their final points. This then implies that there exists a sufficiently small $\gamma>0$ such that $t_{j+1}$ is the unique zero of $u$ defined by (11) in the interval $\left(t_{j+1}-\gamma, t_{j+1}+\gamma\right)$. Due to (15), the function

$$
\tilde{u}(t)=S(t, \tilde{x}(t))
$$

will also change sign in $\left(t_{j+1}-\gamma, t_{j+1}+\gamma\right)$ for sufficiently small $\delta, \tau, \beta>0$.

For sufficiently small $\gamma>0$, then

$$
\frac{d}{d t} S(t, x(t)) \neq 0 \text { for all } t \in\left(t_{j+1}-\gamma, t_{j+1}+\gamma\right)
$$

and the estimate

$$
\begin{aligned}
\left\|\frac{d}{d t} S(t, \tilde{x}(t))-\frac{d}{d t} S(t, x(t))\right\| & =\left\|S_{t}(t, \tilde{x}(t))+S_{x}(t, \tilde{x}(t)) \dot{\tilde{x}}(t)-S_{t}(t, x(t))-S_{x}(t, x(t)) \dot{x}(t)\right\| \\
\leq & \left\|S_{t}(t, \tilde{x}(t))-S_{t}(t, x(t))\right\|+\left\|S_{x}(t, \tilde{x}(t))(\dot{\tilde{x}}(t)-f(t, \tilde{x}(t)))\right\| \\
& +\left\|S_{x}(t, \tilde{x}(t)) f(t, \tilde{x}(t))-S_{x}(t, x(t)) \dot{x}(t)\right\| \\
\leq & D(\|\tilde{x}(t)-x(t)\|+\beta)
\end{aligned}
$$

holds for some constant $D>0$. This shows that

$$
\frac{d}{d t} S(t, \tilde{x}(t)) \neq 0 \text { for all } t \in\left(t_{j+1}-\gamma, t_{j+1}+\gamma\right)
$$

for sufficiently small $\delta, \tau, \beta>0$. Hence, the function $\tilde{u}$ from (16) has a unique zero in the interval $\left(t_{j+1}-\gamma, t_{j+1}+\gamma\right)$.

We now assume that $\tilde{t}_{j+1} \in\left(t_{j+1}-\gamma, t_{j+1}+\gamma\right)$ and that $\sigma>0$ is sufficiently small. Then we consider the nonlinear system of equations

$$
\hat{S}\left(t, \hat{t}_{j+1}, \hat{x}_{j+1}, \hat{\sigma}\right)=0
$$

defined by

$$
\hat{S}\left(t, \hat{t}_{j+1}, \hat{x}_{j+1}, \hat{\sigma}\right)=S\left(t, x\left(t, \hat{t}_{j+1}, \hat{x}_{j+1}\right)\right)-\hat{\sigma},
$$

where $x\left(t, \hat{t}_{j+1}, \hat{x}_{j+1}\right)$ denotes the (local) solution (10) satisfying the initial condition $x\left(\hat{t}_{j+1}\right)=$ $\hat{x}_{j+1}$.

Obviously, the function $\hat{S}$ is defined in a neighborhood of $\left(t_{j+1}, \tilde{t}_{j+1}, x\left(\tilde{t}_{j+1}\right), 0\right)$ and satisfies

(a) $\hat{S}\left(t_{1}, \tilde{t}_{j+1}, x\left(\tilde{t}_{j+1}\right), 0\right)=S\left(t_{j+1}, x\left(t_{j+1}, \tilde{t}_{j+1}, x_{1}\left(\tilde{t}_{j+1}\right)\right)\right)=S\left(t_{j+1}, x\left(t_{j+1}\right)\right)=0$,

(b) $\frac{\partial}{\partial t} \hat{S}\left(t_{j+1}, \tilde{t}_{j+1}, x\left(\tilde{t}_{j+1}\right), 0\right)=S_{t}\left(t_{j+1}, x\left(t_{j+1}\right)\right)+S_{x}\left(t_{j+1}, x\left(t_{j+1}\right)\right) \dot{x}\left(t_{j+1}\right) \neq 0$.

Thus, we can apply the implicit function theorem and we obtain that locally there exists a function $Z$ which is as smooth as $\hat{S}$ and satisfies

(a) $\quad t_{j+1}=Z\left(\tilde{t}_{j+1}, x\left(\tilde{t}_{j+1}\right), 0\right)$,

(b) $\hat{S}\left(Z\left(\hat{t}_{j+1}, \hat{x}_{j+1}, \hat{\sigma}\right), \hat{t}_{j+1}, \hat{x}_{j+1}, \hat{\sigma}\right)=0$ for all $\left(\hat{t}_{j+1}, \hat{x}_{j+1}, \hat{\sigma}\right)$.

Since

$$
\begin{aligned}
& \hat{S}\left(\tilde{t}_{j+1}, \tilde{t}_{j+1}, \tilde{x}\left(\tilde{t}_{j+1}\right), S\left(\tilde{t}_{j+1}, \tilde{x}\left(\tilde{t}_{j+1}\right)\right)\right) \\
& \quad=S\left(\tilde{t}_{j+1}, x\left(\tilde{t}_{j+1}, \tilde{t}_{j+1}, \tilde{x}\left(\tilde{t}_{j+1}\right)\right)-S\left(\tilde{t}_{j+1}, \tilde{x}\left(\tilde{t}_{j+1}\right)\right)=0\right.
\end{aligned}
$$

within the validity of the implicit function theorem for sufficiently small $\delta, \tau, \beta, \sigma>0$, we conclude that

$$
\tilde{t}_{j+1}=Z\left(\tilde{t}_{j+1}, \tilde{x}\left(\tilde{t}_{j+1}\right), S\left(\tilde{t}_{j+1}, \tilde{x}\left(\tilde{t}_{j+1}\right)\right)\right) .
$$

Hence

$$
\begin{aligned}
\left|\tilde{t}_{j+1}-t_{j+1}\right| & =\left|Z\left(\tilde{t}_{j+1}, \tilde{x}\left(\tilde{t}_{j+1}\right), S\left(\tilde{t}_{j+1}, \tilde{x}\left(\tilde{t}_{j+1}\right)\right)\right)-Z\left(\tilde{t}_{j+1}, x\left(\tilde{t}_{j+1}\right), 0\right)\right| \\
& \leq K\left(\left\|\tilde{x}\left(\tilde{t}_{j+1}\right)-x\left(\tilde{t}_{j+1}\right)\right\|+\sigma\right)
\end{aligned}
$$


with some constant $K>0$. Finally, in the same way as in (13), we get

$$
\begin{aligned}
\left\|\tilde{x}\left(\tilde{t}_{j+1}\right)-x\left(t_{j+1}\right)\right\| & \leq\left\|\tilde{x}\left(\tilde{t}_{j+1}\right)-x\left(\tilde{t}_{j+1}\right)\right\|+\left\|x\left(\tilde{t}_{j+1}\right)-x\left(t_{j+1}\right)\right\| \\
& \leq\left\|\tilde{x}\left(\tilde{t}_{j+1}\right)-x\left(\tilde{t}_{j+1}\right)\right\|+M\left|\tilde{t}_{j+1}-t_{j+1}\right| .
\end{aligned}
$$

We summarize this analysis in the following stability estimate for a single mode in a switched system of ODEs.

Lemma 11 Let all assumptions in the above construction for a single mode of a hybrid system of ODEs be satisfied. Then, for sufficiently small $\delta, \tau, \beta, \sigma>0$ there exist constants $\kappa_{\delta}, \kappa_{\tau}, \kappa_{\beta}, \kappa_{\sigma}>0$ such that

$$
\left\|\tilde{x}\left(\tilde{t}_{j+1}\right)-x\left(t_{j+1}\right)\right\|+\left|\tilde{t}_{j+1}-t_{j+1}\right| \leq \kappa_{\delta} \delta+\kappa_{\tau} \tau+\kappa_{\beta} \beta+\kappa_{\sigma} \sigma .
$$

Remark 12 The unique zero in $\left(t_{j+1}-\gamma, t_{j+1}+\gamma\right)$ of $\tilde{u}$ from (16), however, does not need to be the first zero of $\tilde{u}$ beyond $\tilde{t}_{j}$. Due to the perturbations it is possible that there exists a zero immediately after $\tilde{t}_{j}$, typically together with the observation that the given initial state seems to be infeasible. If this is the case, then the implementation of the zero finder for the switching points must take care to skip such an artificial switching point in order to obtain a robust solver for hybrid systems.

To extend Lemma 11 from a single regular arc to several regular arcs, we now assume that $\mathbf{x}=\left(x_{0}^{j_{0}}, x_{1}^{j_{1}}, \ldots, x_{N-1}^{j_{N-1}}\right)$ represents a regular solution of a hybrid system of ODEs and that $\tilde{\mathbf{x}}=\left(\tilde{x}_{0}^{j_{0}}, \tilde{x}_{1}^{j_{1}}, \ldots, \tilde{x}_{N-1}^{j_{N-1}}\right)$ is an approximate solution. In particular, we assume that the same switching structure is valid, i. e., that we have the same modes for $\mathbf{x}$ and $\tilde{\mathbf{x}}$, but instead of switching points $\mathbf{t}=\left(t_{0}, t_{1}, \cdots, t_{N}\right)$ for $\mathbf{x}$ we may have (possibly) perturbed switching points $\tilde{\mathbf{t}}=\left(\tilde{t}_{0}, \tilde{t}_{1}, \cdots, \tilde{t}_{N}\right)$ for $\tilde{\mathbf{x}}$.

For the transitions, we assume that

$$
\begin{aligned}
& \left(t_{i+1}, x_{i+1}^{j_{i+1}}\left(t_{i+1}\right)\right)=T\left(t_{i+1}, x_{i}^{j_{i}}\left(t_{i+1}\right)\right), \\
& \left\|\left(\tilde{t}_{i+1}, \tilde{x}_{i+1}^{j_{i+1}}\left(\tilde{t}_{i+1}\right)\right)-T\left(\tilde{t}_{i+1}, \tilde{x}_{i}^{j_{i}}\left(\tilde{t}_{i+1}\right)\right)\right\| \leq \varrho, \quad i=0, \ldots, N-2
\end{aligned}
$$

where for simplicity we have omitted the (uniquely fixed) lower index of the transition functions. If the transition functions are Lipschitz continuous, then we obtain that

$$
\begin{aligned}
& \left\|\left(\tilde{t}_{i+1}, \tilde{x}_{i+1}^{j_{i+1}}\left(\tilde{t}_{i+1}\right)\right)-\left(t_{i+1}, x_{i+1}^{j_{i+1}}\left(t_{i+1}\right)\right)\right\| \\
& \quad=\left\|\left(\tilde{t}_{i+1}, \tilde{x}_{i+1}^{j_{i+1}}\left(\tilde{t}_{i+1}\right)\right)-T\left(\tilde{t}_{i+1}, \tilde{x}_{i}^{j_{i}}\left(\tilde{t}_{i+1}\right)\right)\right\| \\
& \quad+\left\|T\left(\tilde{t}_{i+1}, \tilde{x}_{i}^{j_{i}}\left(\tilde{t}_{i+1}\right)\right)-T\left(t_{i+1}, x_{i}^{j_{i}}\left(t_{i+1}\right)\right)\right\| \\
& \leq \varrho+U\left\|\left(\tilde{t}_{i+1}, \tilde{x}_{i}^{j_{i}}\left(\tilde{t}_{i+1}\right)\right)-\left(t_{i+1}, x_{i}^{j_{i}}\left(t_{i+1}\right)\right)\right\|
\end{aligned}
$$

with some constant $U>0$. Combining inductively the estimates (18) for every transition with the estimate (17) for every regular arc, we obtain

$$
\left\|\left(\tilde{t}_{N}, \tilde{x}_{N-1}^{j_{N-1}}\left(\tilde{t}_{N}\right)\right)-\left(t_{N}, x_{N-1}^{j_{N-1}}\left(t_{N}\right)\right)\right\| \leq \kappa_{\delta} \delta+\kappa_{\tau} \tau+\kappa_{\beta} \beta+\kappa_{\sigma} \sigma+\kappa_{\varrho} \varrho .
$$

for sufficiently small $\delta, \tau, \beta, \sigma, \varrho>0$ with suitable constants $\kappa_{\delta}, \kappa_{\tau}, \kappa_{\beta}, \kappa_{\sigma}, \kappa_{\varrho}>0$.

Assuming that the initial and final position are not subject to perturbations, as they typically are given as data, we are allowed to set $\tau=0$ and it remains to estimate the perturbation of the final state.

Lemma 13 Let all assumptions in the above construction for a hybrid system of ODEs be satisfied. Then for sufficiently small $\delta, \beta, \sigma, \varrho>0$, there exist constants $\kappa_{\delta}, \kappa_{\beta}, \kappa_{\sigma}, \kappa_{\varrho}>0$ such that

$$
\left\|\tilde{x}^{j_{N-1}}\left(t_{N}\right)-x^{j_{N-1}}\left(t_{N}\right)\right\| \leq \kappa_{\delta} \delta+\kappa_{\beta} \beta+\kappa_{\sigma} \sigma+\kappa_{\varrho} \varrho .
$$


Having obtained a stability estimate for hybrid ODEs, we can easily extend these estimates to regular hybrid systems of DAEs.

For strangeness-free DAEs in the reduced formulation (8), we use (12c) and assume that

$$
\begin{array}{ll}
\text { (a) } & \left\|\dot{\tilde{x}}_{1}^{j}(t)-\mathcal{L}\left(t, \tilde{x}_{1}^{j}(t)\right)\right\| \leq \beta, \quad \text { for all } t \in\left[\tilde{t}_{j}, \tilde{t}_{j+1}\right], \\
\text { (b) }\left\|\tilde{x}_{2}^{j}(t)-\mathcal{R}\left(t, \tilde{x}_{1}^{j}(t)\right)\right\| \leq \eta,
\end{array}
$$

for every regular arc, together with all above assumptions for the (decoupled) ordinary differential equation for $x_{1}^{j}$ including Lipschitz continuity for all involved functions.

Since the transformation $Q$ in (7) is only used for a theoretical reformulation of the problem to state the arising perturbations as in (19), it is not subject to computational errors. Since the computation of the algebraic part, given here as $x_{2}^{j}$, is not to subject to a propagation of the error, we only need to transform the initial value to get the initial value of the differential part, apply the above results for hybrid systems of ODEs to the differential part, and then consider (19b) at the end point in order to determine the final algebraic part and final solution by transforming back according to (7). Since the involved transformations (7) are linear with bounded norm and since

$$
\begin{aligned}
& \left\|\tilde{x}_{2, N-1}^{j_{N-1}}\left(t_{N}\right)-x_{2, N-1}^{j_{N-1}}\left(t_{N}\right)\right\| \\
& \quad \leq\left\|\tilde{x}_{2, N-1}^{j_{j-1}}\left(t_{N}\right)-\mathcal{R}\left(t_{N}, \tilde{x}_{1, N-1}(t)\right)\right\|+\left\|\mathcal{R}\left(t_{N}, \tilde{x}_{1, N-1}\left(t_{N}\right)\right)-\mathcal{R}\left(t_{N}, x_{1, N-1}\left(t_{N}\right)\right)\right\| \\
& \quad \leq \eta+R\left\|\tilde{x}_{1, N-1}\left(t_{N}\right)-x_{1, N-1}\left(t_{N}\right)\right\|
\end{aligned}
$$

with some constant $R>0$, Lipschitz continuity is maintained and we obtain the following result.

Theorem 14 Let all assumptions in the above construction for a hybrid system of DAEs be satisfied. Then, for sufficiently small $\delta, \beta, \eta, \sigma, \varrho, \eta>0$, there exist constants $\kappa_{\delta}, \kappa_{\beta}, \kappa_{\eta}, \kappa_{\sigma}, \kappa_{\varrho}, \kappa_{\eta}>0$ such that

$$
\left\|\tilde{x}^{j_{N-1}}\left(t_{N}\right)-x^{j_{N-1}}\left(t_{N}\right)\right\| \leq \kappa_{\delta} \delta+\kappa_{\tau} \tau+\kappa_{\beta} \beta+\kappa_{\eta} \eta+\kappa_{\sigma} \sigma+\kappa_{\varrho} \varrho+\kappa_{\eta} \eta .
$$

Remark 15 If we assume that there are no computational errors, i. e., if $\beta=\eta=\sigma=\varrho=\eta=0$, then the estimate (20) reduces to

$$
\left\|\tilde{x}^{j_{N-1}}\left(t_{N}\right)-x^{j_{N-1}}\left(t_{N}\right)\right\| \leq \kappa_{\delta} \delta
$$

for sufficiently small $\delta>0$, cp. Theorem 7 .

In this section we have obtained stability estimates for hybrid ODEs and extended the results to regular strangeness-free hybrid DAEs. There is, however, the immediate question what happens in the case that a hybrid system of DAEs loses regularity. In this case regularization techniques are necessary. We discuss this issue in the next section.

\section{Non-regular behavior}

In this section we discuss hybrid DAEs which have a lack of regularity. In view of Definition 3, such a lack of regularity can have several reasons which include at least the following cases:

1. more than one switching condition is satisfied;

2. the switching condition has a non-regular solution;

3. there is no finite collection of switching points;

4. the flow does not lead to $\left(S_{l}^{j} \circ \Phi_{t, \underline{t}}^{j}\right)\left(\underline{t}, \underline{z}^{j}\right)>0$ for $t \in\left(t_{j}, t_{j}+\varepsilon\right]$ for all $l \in\left\{1, \ldots, L^{j}\right\}$, since

(a) the flow is in at least one of the switching surfaces, i. e., we have that $\left(S_{l}^{j} \circ \Phi_{t, \underline{t}}^{j}\right)\left(\underline{t}, \underline{z}^{j}\right)=0$ for $t \in\left(t_{j}, t_{j}+\varepsilon\right]$ and some $l \in\left\{1, \ldots, L^{j}\right\}$, 
(b) we have instantaneous further switching, i. e., we have infeasibility due to $\left(S_{l}^{j} \circ \Phi_{t, \underline{t}}^{j}\right)\left(\underline{t}, \underline{z}^{j}\right)<$ 0 for $t \in\left(t_{j}, t_{j}+\varepsilon\right]$ and some $l \in\left\{1, \ldots, L^{j}\right\}$.

Since a numerical treatment of hybrid DAEs with a non-regular solution makes no sense, it is necessary to perform a regularization by remodeling the given problem. Some of the discussed cases of non-regularity allow for ad hoc regularization techniques, but observe that these techniques may be non-physical, since they are not based on the physical background, but they are needed for the sensible numerical integration and they may also give an indication for a possible reformulation on physical grounds.

\subsection{Regularization techniques}

In the following, we separately discuss various regularization techniques, but it should be noted that these cases may occur simultaneously, so that it may be necessary to apply several of these together in order to obtain a regularization.

1. Non-unique switching condition. If the end point $\left(t_{j+1}, x^{j}\left(t_{j+1}\right)\right)$ satisfies more than one switching condition, then we can simply replace the current mode by selecting one of the activated switching conditions and omitting the others. The particular choice may be viewed as a dedicated hierarchy in the switching conditions.

2. Non-regular solution of the switching condition. If the solution of the equation for the switching condition is non-regular, then small perturbations can lead to a different change of modes. A well-known example of this case is the modeling of billiard [6,12], when a moving ball just touches another ball. For this example, we know that the overall solution depends smoothly on the initial condition but that the condition number of the Jacobian that determines the switching point may be very large, especially when there are several hits. In the general case, however, there seems to be no good ad hoc strategy for regularizing the problem.

3. Infinite number of switching points. It may happen that an infinite number of switching points occurs in the given integration interval. Since we have assumed this interval to be compact, an infinite number of switching points implies that there is at least one accumulation point in the set of switching points. A prominent example for this case is the bouncing ball [6, 12], when the ball loses a certain percentage of its momentum with every bounce. A simple regularization strategy in this case is to characterize the behavior of the physical system after the accumulation point by a mode and to switch to this mode a sufficiently small amount of time before we reach the accumulation point.

4.(a) Flow in switching surface. In the case that the flow is in the switching surface, we have that

$$
\left(S_{l}^{j} \circ \Phi_{t, t_{j}}^{j}\right)\left(t_{j}, z_{0}^{j}\right)=0 \quad \text { for all } t \in\left[t_{j}, t_{j}+\varepsilon\right]
$$

holds for one or more $l \in\left\{1, \ldots, L^{j}\right\}$. But this means nothing else than that these $S_{l}^{j}$ are first integrals of the DAE of mode $j$. Thus, we actually have an overdetermined but consistent system of DAEs of the form

$$
\begin{aligned}
F^{j}\left(t, x^{j}, \dot{x}^{j}\right) & =0, \\
S^{j}\left(t, x^{j}\right) & =0,
\end{aligned}
$$

where $S^{j}$ gathers all the first integrals among the switching functions. To study the properties of this system, we go over to the formulation (9) so that (21) takes the form

$$
\begin{aligned}
\dot{x}_{1}^{j} & =\mathcal{L}^{j}\left(t, x_{1}^{j}\right), \\
x_{2}^{j} & =\mathcal{R}^{j}\left(t, x_{1}^{j}\right), \\
0 & =\mathcal{S}^{j}\left(t, x_{1}^{j}, x_{2}^{j}\right) .
\end{aligned}
$$


Thus, we have additional constraints of the form

$$
\mathcal{S}^{j}\left(t, x_{1}^{j}, \mathcal{R}^{j}\left(t, x_{1}^{j}\right)\right)=0
$$

which may in parts be trivial equations $0=0$. Of course, these redundant equations can be simply omitted. Assuming then that $\frac{d}{d x_{1}^{j}} \mathcal{S}^{j}$ has full row rank, we can solve (22) for some of the components of $x_{1}$. For these components, the corresponding differential equations contained in $\dot{x}_{1}^{j}=\mathcal{L}^{j}\left(t, x_{1}^{j}\right)$ are redundant and can be omitted. If we denote by $\mathcal{P}^{j}$ the projector which selects the components of $x_{1}^{j}$ that are not fixed by (22), then we get a DAE of the form

$$
\begin{aligned}
\mathcal{P}^{j} \dot{x}_{1}^{j} & =\mathcal{P}^{j} \mathcal{L}^{j}\left(t, x_{1}^{j}\right), \\
x_{2}^{j} & =\mathcal{R}^{j}\left(t, x_{1}^{j}\right), \\
0 & =\mathcal{S}^{j}\left(t, x_{1}^{j}, x_{2}^{j}\right) .
\end{aligned}
$$

The original mode $j$ then should be replaced by the regular DAE (23), together with the switching functions which were not first integrals of the original DAE. Of course, the actual construction should and can be performed on the original (possibly higher-index) formulation of the mode. For corresponding details, see [19].

4.(b) Instantaneous further switching. The case of instantaneous further switching is characterized by the observation that

$$
\left(S_{l}^{j} \circ \Phi_{t, t_{j}}^{j}\right)\left(t_{j}, x^{j}\left(t_{j}\right)\right)<0 \quad \text { for all } t \in\left(t_{j}, t_{j}+\varepsilon\right]
$$

holds for one or more $l \in\left\{1, \ldots, L^{j}\right\}$. Similar to the discussion in Case 1., we then must select one of the activated switching conditions, say $\hat{l}^{j}$, in order to fix a unique new mode for the system. If we denote the activated switching condition of mode $k$ which has led to the current mode $j$ by $\hat{l}^{k}$, then we can replace mode $k$ by a new mode consisting of the same DAE and switching functions, but replacing $\sigma^{k}\left(\hat{l}^{k}\right)=j$ by $\sigma^{k}\left(\hat{l}^{k}\right)=m$, where $m=\sigma^{j}\left(\hat{l}^{j}\right)$, and the transition function $T_{\hat{l}^{k}}^{j, k}$ by $T_{\hat{l}^{k}}^{m, k} \circ \Phi_{t_{j}, t_{j}}^{j} \circ T_{\hat{l}^{k}}^{j, k}$.

The discussed regularization techniques may have to be iterated to deal with systems where several of the described non-regularities arise. In particular, there may be several instantaneous further switchings one after the other and one must hope that this terminates after a finite number of times. It is, however, possible that this iteration actually leads to an infinite loop. One such case is chattering which is discussed in the next subsection.

\subsection{Chattering}

Chattering is the phenomenon that we just switch back to the previous mode and the previous state requires to switch again to the current system. This frequently happens in actual physical systems, see e. g., [15, 22, 24], and in this case it is necessary to modify the model to incorporate the chattering effect. On the other hand, even correctly modeled chattering typically leads to systems with highly oscillatory solutions. For such systems the numerical solution usually is very difficult and costly. Since one is often not really interested in resolving the chattering but in the more global behavior of the systems, it is also of interest to develop regularization techniques especially for this case.

Since it is sufficient for the discussion of chattering to consider only two modes and the specific switching conditions which are responsible for the chattering, we assume that the hybrid system is given by the two DAEs

$$
F^{1}\left(t, x^{1}, \dot{x}^{1}\right)=0, \quad F^{2}\left(t, x^{2}, \dot{x}^{2}\right)=0,
$$

which we may assume to be already strangeness-free, the switching functions $S^{1}$ and $S^{2}$, and transition functions

$$
T^{21}: \mathbb{M}^{1} \rightarrow \mathbb{L}_{0}^{2}, \quad T^{12}: \mathbb{M}^{2} \rightarrow \mathbb{L}_{0}^{1},
$$




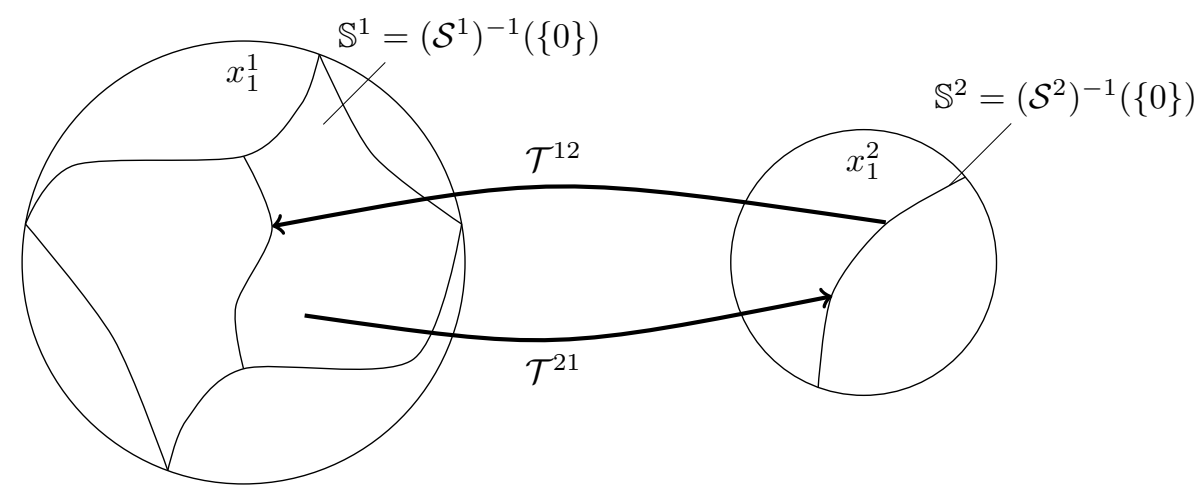

Figure 1: Occurence of chattering

where $\mathbb{M}^{1}=\left(S^{1}\right)^{-1}(\{0\})$ and $\mathbb{M}^{2}=\left(S^{2}\right)^{-1}(\{0\})$.

Let $\hat{T}^{21}: \mathbb{M}^{1} \rightarrow \mathbb{M}^{2}$ and $\hat{T}^{12}: \mathbb{M}^{2} \rightarrow \mathbb{M}^{1}$ be defined by the following implications

$$
\begin{aligned}
& T^{21}\left(t, x^{1}\right)=\left(t, x^{2}, \dot{x}^{2}\right) \quad \Longrightarrow \quad \hat{T}^{21}\left(t, x^{1}\right)=\left(t, x^{2}\right), \\
& T^{12}\left(t, x^{2}\right)=\left(t, x^{1}, \dot{x}^{1}\right) \quad \Longrightarrow \quad \hat{T}^{12}\left(t, x^{2}\right)=\left(t, x^{1}\right) .
\end{aligned}
$$

Then the effect of chattering is characterized by the conditions

$$
\begin{array}{ll}
\hat{T}^{21}\left(\mathbb{M}^{1}\right) \subseteq \mathbb{M}^{2}, & \hat{T}^{12}\left(\mathbb{M}^{2}\right) \subseteq \mathbb{M}^{1} \\
\frac{\partial}{\partial t} S^{1}+\frac{\partial}{\partial x^{1}} S^{1} \dot{x}^{1}<0, & \frac{\partial}{\partial t} S^{2}+\frac{\partial}{\partial x^{2}} S^{2} \dot{x}^{2}<0,
\end{array}
$$

where the sign conditions hold on the switching surfaces in $\mathbb{L}_{0}^{1}$ and $\mathbb{L}_{0}^{2}$ belonging to the switching conditions that are responsible for the chattering, and by

$$
\hat{T}^{21} \circ \hat{T}^{12} \circ \hat{T}^{21}=\hat{T}^{21}, \quad \hat{T}^{12} \circ \hat{T}^{21} \circ \hat{T}^{12}=\hat{T}^{12} .
$$

This means that $\hat{T}^{21}$ is an inner and outer inverse of $\hat{T}^{12}$ and vice versa, $[5,8]$.

A very simple method to regularize such a hybrid system is to introduce hysteresis [2] into the system by moving the switching surface in such a way that the transition functions lead to the interior of the feasible region, for example by choosing some small $\delta>0$, replacing the switching conditions by

$$
S^{1}\left(t, x^{1}\right)+\delta=0, \quad S^{2}\left(t, x^{2}\right)+\delta=0,
$$

and taking care that the transition functions still map to $\mathbb{M}^{1}$ and $\mathbb{M}^{2}$, see the numerical experiments below.

A second method to deal with chattering is the so-called Filippov regularization, see [11], in the case that the two modes and the switching surfaces coincide. The idea of Filippov regularization is to take the unique convex combination of the two flows which yields a flow that is restricted to the common switching surface. Actually, this corresponds to the introduction of a new mode into the original hybrid system together with suitable switching conditions and transfer functions.

Being originally developed for hybrid ODE models and generalized to hybrid DAEs in [15, 24, 25], we generalize this concept further to the case that the variables of the involved models do not coincide.

For the construction, we first discuss the (autonomous) decoupled case, with $d^{1}$ differential and $a^{1}$ algebraic equations in the first mode and $d^{2}$ differential and $a^{2}$ algebraic equations in the second mode,

$$
\begin{array}{llll}
\left(d^{1} \text { eq. }\right) & \dot{x}_{1}^{1}=\mathcal{L}^{1}\left(x_{1}^{1}\right), & \left(d^{2} \text { eq. }\right) & \dot{x}_{1}^{2}=\mathcal{L}^{2}\left(x_{1}^{2}\right), \\
\left(a^{1} \text { eq. }\right) & x_{2}^{1}=\mathcal{R}^{1}\left(x_{1}^{1}\right), & \left(a^{2} \text { eq. }\right) & x_{2}^{2}=\mathcal{R}^{2}\left(x_{1}^{2}\right),
\end{array}
$$


cp. Figure 5.2, and we assume without loss of generality that $d^{1} \geq d^{2}$.

Chattering occurs at the time point $t$ if

$$
\left(\mathcal{T}^{21} \circ \mathcal{T}^{12}\right)\left(x_{1}^{2}(t)\right)=x_{1}^{2}(t)
$$

and the conditions

$$
\begin{array}{ll}
\left(\mathcal{S}^{2} \circ \mathcal{T}^{12}\right)\left(x_{1}^{2}(t)\right)=0, & \mathcal{S}^{2}\left(x_{1}^{2}(t)\right)=0, \\
\mathcal{S}_{x_{1}^{1}}^{1}\left(\mathcal{T}^{12}\left(x_{1}^{2}(t)\right)\right) \mathcal{L}^{1}\left(\mathcal{T}^{12}\left(x_{1}^{2}(t)\right)\right)<0, & \mathcal{S}_{x_{1}^{2}}^{2}\left(x_{1}^{2}(t)\right) \mathcal{L}^{2}\left(x_{1}^{2}(t)\right)<0
\end{array}
$$

are satisfied, where the lower index as in $\mathcal{S}_{x_{1}^{1}}^{1}$ means the derivative with respect to $x_{1}^{1}$.

A possible regularization would be to restrict the flows to the switching surface. For the second mode this leads to

$$
\begin{aligned}
& \text { (d } d^{2} \text { eq.) } \dot{x}_{1}^{2}=\mathcal{L}^{2}\left(x_{1}^{2}\right), \\
& \text { (a } a^{2} \text { eq.) } x_{2}^{2}=\mathcal{R}^{2}\left(x_{1}^{2}\right), \\
& \text { (1 eq.) } \quad 0=\mathcal{S}^{2}\left(x_{1}^{2}\right),
\end{aligned}
$$

which is an over-determined and inconsistent system. In particular, it is not directly evident what would be a meaningful flow on the manifold defined by the algebraic constraints in (29).

Observing that the differentiation of the transition relation

$$
x_{1}^{2}=\mathcal{T}^{12}\left(x_{1}^{1}\right)
$$

yields

$$
\dot{x}_{1}^{2}=\mathcal{T}_{x_{1}^{1}}^{21}\left(x_{1}^{1}\right) \dot{x}_{1}^{1}=\mathcal{T}_{x_{1}^{1}}^{21}\left(\mathcal{T}^{12}\left(x_{1}^{2}\right)\right) \mathcal{L}^{1}\left(\mathcal{T}^{12}\left(x_{1}^{2}\right)\right),
$$

the idea of Filippov regularization in the ODE case is to combine the two proposed flows from (29) and (30) to a possible flow on the constraint manifold of (29).

In this way, we obtain the DAE

$$
\begin{array}{ll} 
& w_{1}^{1}=\mathcal{T}_{x_{1}^{1}}^{21}\left(\mathcal{T}^{12}\left(x_{1}^{2}\right)\right) \mathcal{L}^{1}\left(\mathcal{T}^{12}\left(x_{1}^{2}\right)\right), \\
& w_{1}^{2}=\mathcal{L}^{2}\left(x_{1}^{2}\right) \\
\left(d^{2} \text { eq. }\right) & \dot{x}_{1}^{2}=\alpha w_{1}^{1}+(1-\alpha) w_{1}^{2} \\
\text { (a } \left.a^{2} \text { eq. }\right) & x_{2}^{2}=\mathcal{R}^{2}\left(x_{1}^{2}\right), \\
\text { (1 eq. }) & 0=\mathcal{S}^{2}\left(x_{1}^{2}\right)
\end{array}
$$

for the unknowns $\left(x_{1}^{2}, x_{2}^{2}, \alpha\right)$, if we assume that the quantities $w_{1}^{1}, w_{1}^{2}$ are eliminated. In particular, we only need to consider the DAE

$$
\begin{array}{ll}
\text { (d } \left.d^{2} \text { eq. }\right) & \dot{x}_{1}^{2}=\alpha \mathcal{T}_{x_{1}^{1}}^{21}\left(\mathcal{T}^{12}\left(x_{1}^{2}\right)\right) \mathcal{L}^{1}\left(\mathcal{T}^{12}\left(x_{1}^{2}\right)\right)+(1-\alpha) \mathcal{L}^{2}\left(x_{1}^{2}\right), \\
(1 \text { eq. }) & 0=\mathcal{S}^{2}\left(x_{1}^{2}\right)
\end{array}
$$

for $\left(x_{1}^{2}, \alpha\right)$.

Note that (32) is a semi-explicit DAE of the form

$$
\dot{x}=f(x, y), \quad 0=g(x) .
$$

It is well-known that such a DAE cannot be strangeness-free, but it satisfies Hypothesis 1 with $\mu=1$ if $g_{x}(x) f_{y}(x, y)$ is nonsingular along the solution.

To deal with this situation we assume that regularization with the help of hysteresis as described above is possible. In particular, we assume that extending the solution a little bit beyond the switching surface in one mode and then switching to the other mode yields a point which is feasible with respect to the switching condition in this mode, i. e.,

$$
\begin{aligned}
& \mathcal{S}_{x_{1}^{2}}^{2}\left(\mathcal{T}^{21}\left(x_{1}^{1}(t)\right)\right) \mathcal{T}_{x_{1}^{1}}^{21}\left(x_{1}^{1}(t)\right) \mathcal{L}^{1}\left(x_{1}^{1}(t)\right)>0 \\
& \mathcal{S}_{x_{1}^{1}}^{1}\left(\mathcal{T}^{12}\left(x_{1}^{2}(t)\right)\right) \mathcal{T}_{x_{1}^{2}}^{12}\left(x_{1}^{2}(t)\right) \mathcal{L}^{2}\left(x_{1}^{2}(t)\right)>0 .
\end{aligned}
$$


In (32) the quantity $g_{x}(x) f_{y}(x, y)$ is a scalar given by

$$
\gamma=\mathcal{S}_{x_{1}^{2}}^{2}\left(x_{1}^{2}\right)\left[\mathcal{T}_{x_{1}^{1}}^{21}\left(\mathcal{T}^{12}\left(x_{1}^{2}\right)\right) \mathcal{L}^{1}\left(\mathcal{T}^{12}\left(x_{1}^{2}\right)\right)-\mathcal{L}^{2}\left(x_{1}^{2}\right)\right] .
$$

Setting $x_{1}^{1}=\mathcal{T}^{12}\left(x_{1}^{2}\right)$ and using (27), which gives $\mathcal{T}^{21}\left(x_{1}^{1}\right)=x_{1}^{2}$, we obtain that

$$
\gamma=\mathcal{S}_{x_{1}^{2}}^{2}\left(\mathcal{T}^{21}\left(x_{1}^{1}\right)\right) \mathcal{T}_{x_{1}^{1}}^{21}\left(x_{1}^{1}\right) \mathcal{L}^{1}\left(x_{1}^{1}\right)-\mathcal{S}_{x_{1}^{2}}^{2}\left(x_{1}^{2}\right) \mathcal{L}^{2}\left(x_{1}^{2}\right)
$$

and this gives

$$
\gamma>0
$$

due to (28) and (33). Hence, the DAE (31) and so the DAE (32) satisfy Hypothesis 1 with $\mu=1$.

The corresponding hidden constraint is given by

$$
\mathcal{S}_{x_{1}^{2}}^{2}\left(x_{1}^{2}\right)\left[\alpha \mathcal{T}_{x_{1}^{1}}^{21}\left(\mathcal{T}^{12}\left(x_{1}^{2}\right)\right) \mathcal{L}^{1}\left(\mathcal{T}^{12}\left(x_{1}^{2}\right)\right)+(1-\alpha) \mathcal{L}^{2}\left(x_{1}^{2}\right)\right]=0 .
$$

Utilizing (34), we can solve (35) for $\alpha$ to obtain

$$
\alpha=\frac{-\mathcal{S}_{x_{1}^{2}}^{2}\left(x_{1}^{2}\right) \mathcal{L}^{2}\left(x_{1}^{2}\right)}{\mathcal{S}_{x_{1}^{2}}^{2}\left(x_{1}^{2}\right) \mathcal{T}_{x_{1}^{1}}^{21}\left(\mathcal{T}^{12}\left(x_{1}^{2}\right)\right)-\mathcal{S}_{x_{1}^{2}}^{2}\left(x_{1}^{2}\right) \mathcal{L}^{2}\left(x_{1}^{2}\right)} .
$$

Due to the sign conditions (28) and (33), we see that

$$
\alpha \in(0,1)
$$

Thus we actually use an appropriate convex combination of the involved flows to get a flow on the constraint manifold of (31).

In the non-autonomous case of the form

$$
\begin{aligned}
& \text { (d } \left.d^{1} \text { eq. }\right) \quad \dot{x}_{1}^{1}=\mathcal{L}^{1}\left(t, x_{1}^{1}\right), \quad\left(d^{2} \text { eq. }\right) \quad \dot{x}_{1}^{2}=\mathcal{L}^{2}\left(t, x_{1}^{2}\right), \\
& \text { (a } \left.a^{1} \text { eq.) } \quad x_{2}^{1}=\mathcal{R}^{1}\left(t, x_{1}^{1}\right), \quad \text { (a } a^{2} \text { eq. }\right) \quad x_{2}^{2}=\mathcal{R}^{2}\left(t, x_{1}^{2}\right) \text {, }
\end{aligned}
$$

we must replace the relation (27) by

$$
\left(\mathcal{T}^{21} \circ \mathcal{T}^{12}\right)\left(t, x_{1}^{2}(t)\right)=\left(t, x_{1}^{2}(t)\right)
$$

and, utilizing

$$
\mathcal{T}^{12}\left(t, x_{1}^{2}(t)\right)=\left(t, x_{1}^{1}(t)\right), \quad \frac{d}{d t}\left(\mathcal{T}^{12}\left(t, x_{1}^{2}(t)\right)\right)=\left(1, \dot{x}_{1}^{1}(t)\right),
$$

the relations (28) by

$$
\begin{aligned}
\left(\mathcal{S}^{2} \circ \mathcal{T}^{12}\right)\left(t, x_{1}^{2}(t)\right) & =0 \\
\mathcal{S}_{t}^{1}\left(\mathcal{T}^{12}\left(t, x_{1}^{2}(t)\right)\right)+\mathcal{S}_{x_{1}^{1}}^{1}\left(\mathcal{T}^{12}\left(t, x_{1}^{2}(t)\right)\right) \mathcal{L}^{1}\left(\mathcal{T}^{12}\left(t, x_{1}^{2}(t)\right)\right. & <0 \\
\mathcal{S}^{2}\left(t, x_{1}^{2}(t)\right) & =0 \\
\mathcal{S}_{t}^{2}\left(t, x_{1}^{2}(t)\right)+\mathcal{S}_{x_{1}^{2}}^{2}\left(t, x_{1}^{2}(t)\right) \mathcal{L}^{2}\left(t, x_{1}^{2}(t)\right) & <0
\end{aligned}
$$

The relation for $x_{1}^{2}$ becomes

$$
\dot{x}_{1}^{2}=\Pi_{2}^{2}\left(\mathcal{T}_{t}^{21}\left(\mathcal{T}^{12}\left(t, x_{1}^{2}\right)\right)+\mathcal{T}_{x_{1}^{1}}^{21}\left(\mathcal{T}^{12}\left(t, x_{1}^{2}\right)\right) \mathcal{L}^{1}\left(\mathcal{T}^{12}\left(t, x_{1}^{2}\right)\right)\right),
$$

where $\Pi_{2}^{2}$ denotes the projection onto the second argument of $\left(t, x_{1}^{2}\right)$. The regularized DAE then reads

$$
\begin{array}{ll} 
& \left.w_{1}^{1}=\Pi_{2}^{2}\left(\mathcal{T}_{t}^{21}\left(\mathcal{T}^{12}\left(t, x_{1}^{2}\right)\right)+\mathcal{T}_{x_{1}^{1}}^{21}\left(\mathcal{T}^{12}\left(t, x_{1}^{2}\right)\right) \mathcal{L}^{1}\left(\mathcal{T}^{12}\left(t, x_{1}^{2}\right)\right)\right)\right), \\
& w_{1}^{2}=\mathcal{L}^{2}\left(t, x_{1}^{2}\right), \\
\left(d^{2} \text { eq. }\right) & \dot{x}_{1}^{2}=\alpha w_{1}^{1}+(1-\alpha) w_{1}^{2}, \\
\left(a^{2} \text { eq. }\right) & x_{2}^{2}=\mathcal{R}^{2}\left(t, x_{1}^{2}\right), \\
\text { (1 eq. }) & 0=\mathcal{S}^{2}\left(t, x_{1}^{2}\right) .
\end{array}
$$


Eliminating $w_{1}^{1}, w_{1}^{2}$ yields the relevant part

$$
\begin{array}{ccc}
\left(d^{2} \text { eq. }\right) & \left.\dot{x}_{1}^{2}=\alpha P i_{2}^{2}\left(\mathcal{T}_{t}^{21}\left(\mathcal{T}^{12}\left(t, x_{1}^{2}\right)\right)+\mathcal{T}_{x_{1}^{1}}^{21}\left(\mathcal{T}^{12}\left(t, x_{1}^{2}\right)\right) \mathcal{L}^{1}\left(\mathcal{T}^{12}\left(t, x_{1}^{2}\right)\right)\right)\right) \\
& +(1-\alpha) \mathcal{L}^{2}\left(t, x_{1}^{2}\right), \\
\text { (1 eq. }) & 0=\mathcal{S}^{2}\left(t, x_{1}^{2}\right) . &
\end{array}
$$

The assumptions (33) take the form

$$
\begin{aligned}
\mathcal{S}_{t}^{2}\left(\mathcal{T}^{21}\left(t, x_{1}^{1}(t)\right)\right)+\mathcal{S}_{t, x_{1}^{2}}^{2}\left(\mathcal{T}^{21}\left(t, x_{1}^{1}(t)\right)\right) \mathcal{T}_{x_{1}^{1}}^{21}\left(t, x_{1}^{1}(t)\right) \mathcal{L}^{1}\left(t, x_{1}^{1}(t)\right) & >0 \\
\mathcal{S}_{t}^{1}\left(\mathcal{T}^{12}\left(t, x_{1}^{2}(t)\right)\right) \mathcal{S}_{x_{1}^{1}}^{1}\left(\mathcal{T}^{12}\left(t, x_{1}^{2}(t)\right)\right) \mathcal{T}_{x_{1}^{2}}^{12}\left(t, x_{1}^{2}(t)\right) \mathcal{L}^{2}\left(t, x_{1}^{2}(t)\right) & >0
\end{aligned}
$$

The hidden constraint in (38) is given by

$$
\begin{aligned}
\mathcal{S}_{t}^{2}\left(t, x_{1}^{2}\right)+ & \mathcal{S}_{x_{1}^{2}}^{2}\left(t, x_{1}^{2}\right) \\
& \cdot\left[\alpha \Pi_{2}^{2}\left(\mathcal{T}_{t}^{21}\left(\mathcal{T}^{12}\left(t, x_{1}^{2}\right)\right)+\mathcal{T}_{x_{1}^{1}}^{21}\left(\mathcal{T}^{12}\left(t, x_{1}^{2}\right)\right) \mathcal{L}^{1}\left(\mathcal{T}^{12}\left(t, x_{1}^{2}\right)\right)\right)+(1-\alpha) \mathcal{L}^{2}\left(t, x_{1}^{2}\right)\right] \\
=\alpha[ & \left.\mathcal{S}_{t}^{2}\left(t, x_{1}^{2}\right)+\mathcal{S}_{x_{1}^{2}}^{2}\left(t, x_{1}^{2}\right) \Pi_{2}^{2}\left(\mathcal{T}_{t}^{21}\left(\mathcal{T}^{12}\left(t, x_{1}^{2}\right)\right)+\mathcal{T}_{x_{1}^{1}}^{21}\left(\mathcal{T}^{12}\left(t, x_{1}^{2}\right)\right) \mathcal{L}^{1}\left(\mathcal{T}^{12}\left(t, x_{1}^{2}\right)\right)\right)\right] \\
& (1-\alpha)\left[\mathcal{S}_{t}^{2}\left(t, x_{1}^{2}\right)+\mathcal{S}_{x_{1}^{2}}^{2}\left(t, x_{1}^{2}\right) \mathcal{L}^{2}\left(t, x_{1}^{2}\right)\right] .
\end{aligned}
$$

The term in the first bracket is positive due to (39), and the term in the second bracket is negative due to (37), such that we are in the same situation as in the autonomous case leading to a welldefined $\alpha \in(0,1)$.

In order to lift (36) to the general formulation

$$
\begin{array}{llll}
\text { (d } \left.d^{1} \text { eq. }\right) & \hat{F}_{1}^{1}\left(t, x^{1}, \dot{x}^{1}\right)=0, & \left(d^{2} \text { eq. }\right) & \hat{F}_{1}^{2}\left(t, x^{2}, \dot{x}^{2}\right)=0, \\
\left(a^{1} \text { eq. }\right) & \hat{F}_{2}^{1}\left(t, x^{1}\right)=0, & \left(a^{2} \text { eq. }\right) & \hat{F}_{2}^{2}\left(t, x^{2}\right)=0,
\end{array}
$$

we consider the combined system

$$
\begin{aligned}
& \text { (d } \left.\left.d^{1} \text { eq. }\right) \quad \hat{F}_{1}^{1}\left(t, x^{1}, \dot{w}^{1}\right)=0, \quad \text { (d } d^{2} \text { eq. }\right) \quad \hat{F}_{1}^{2}\left(t, x^{2}, \dot{w}^{2}\right)=0, \\
& {\left[\hat{F}_{2}^{1}\left(t, x^{1}\right)=0,\right] \quad\left[\hat{F}_{2}^{2}\left(t, x^{2}\right)=0,\right]} \\
& \text { (a } a^{1} \text { eq.) } \quad \hat{F}_{2 ; t}^{1}\left(t, x^{1}\right)+\hat{F}_{2 ; x^{1}}^{1}\left(t, x^{1}\right) w^{1}=0 \text {, (a } a^{2} \text { eq.) } \quad \hat{F}_{2 ; t}^{2}\left(t, x^{2}\right)+\hat{F}_{2 ; x^{2}}^{2}\left(t, x^{2}\right) w^{2}=0 \text {, } \\
& {\left[S^{1}\left(t, x^{1}\right)=0,\right] \quad\left[S^{2}\left(t, x^{2}\right)=0,\right]} \\
& \text { ( } \left.n^{1} \text { eq.) }\left(t, x^{1}\right)=T^{12}\left(t, x^{3}\right), \quad \text { ( } n^{2} \text { eq. }\right)\left(t, x^{2}\right)=\left(t, x^{3}\right) \text {, } \\
& \text { ( } n^{3} \text { eq.) } \dot{x}_{3}=\alpha \Pi_{2}^{2}\left(T_{t}^{21}\left(T^{12}\left(t, x^{3}\right)\right)+T_{x^{1}}^{21}\left(T^{12}\left(t, x^{3}\right)\right) w^{1}\right)+(1-\alpha) w^{2} \text {, } \\
& \text { (a } a^{2} \text { eq.) } \hat{F}_{2}^{2}\left(t, x^{3}\right)=0 \\
& \text { (1 eq.) } \quad S^{2}\left(t, x^{3}\right)=0,
\end{aligned}
$$

where the bracketed relations are redundant and can therefore be omitted. Since the matrices

$$
\left[\begin{array}{c}
\hat{F}_{1, \dot{x}^{1}}^{1} \\
\hat{F}_{2, x^{1}}^{1}
\end{array}\right],\left[\begin{array}{c}
\hat{F}_{1, \dot{x}^{2}}^{2} \\
\hat{F}_{2, x^{2}}^{2}
\end{array}\right]
$$

are nonsingular due to Hypothesis 1, the first part of (40) fixes $w^{1} \in \mathbb{R}^{n^{1}}$ and $w^{2} \in \mathbb{R}^{n^{2}}$. The second part of (40) fixes $x^{1} \in \mathbb{R}^{n^{1}}$ and $x^{2} \in \mathbb{R}^{n^{2}}$ in terms of $x^{3} \in \mathbb{R}^{n^{2}}$. The last three relations of (40) finally form an over-determined DAE for $x^{3} \in \mathbb{R}^{n^{2}}$.

If we can show that the flow $\dot{x}_{3}$ is consistent with $\hat{F}_{2}^{2}\left(t, x^{3}\right)=0$, then there are actually only $d^{2}$ relevant relations within the $n^{2}$ equations defining $\dot{x}_{3}$, which would lead to a square system for the unknowns $\left(x^{3}, \alpha\right)$.

Starting from

$$
\begin{aligned}
& \hat{F}_{2, t}^{2}(\left.t, x^{3}\right)+\hat{F}_{2, x^{2}}^{2} \dot{x}^{3}\left(t, x^{3}\right) \\
&= \hat{F}_{2, t}^{2}\left(t, x^{3}\right)+\hat{F}_{2, x^{2}}^{2}\left(t, x^{3}\right) \\
& \quad \cdot\left[\alpha \Pi_{2}^{2}\left(T_{t}^{21}\left(T^{12}\left(t, x^{3}\right)\right)+T_{x^{1}}^{21}\left(T^{12}\left(t, x^{3}\right)\right) w^{1}\right)+(1-\alpha) w^{2}\right] \\
&=\alpha\left[\hat{F}_{2, t}^{2}\left(t, x^{3}\right)+\hat{F}_{2, x^{2}}^{2}\left(t, x^{3}\right) \Pi_{2}^{2}\left(T_{t}^{21}\left(T^{12}\left(t, x^{3}\right)\right)+T_{x^{1}}^{21}\left(T^{12}\left(t, x^{3}\right)\right) w^{1}\right)\right] \\
& \quad+(1-\alpha)\left[\hat{F}_{2, t}^{2}\left(t, x^{3}\right)+\hat{F}_{2, x^{2}}^{2}\left(t, x^{3}\right) w^{2}\right]
\end{aligned}
$$


we see that the second bracket in the last term of (41) vanishes due to (40). Furthermore, since

$$
\hat{F}_{2}^{2}\left(T^{21}\left(t, x^{1}\right)\right) \equiv 0
$$

yields

$$
\begin{aligned}
\hat{F}_{2 ; t}\left(T^{21}\left(t, x^{1}\right)\right)+\hat{F}_{2 ; x^{2}}\left(T^{21}\left(t, x^{1}\right)\right) T_{t}^{21}\left(t, x^{1}\right) & \equiv 0, \\
\hat{F}_{2 ; x^{2}}\left(T^{21}\left(t, x^{1}\right)\right) \Pi_{2}^{2} T_{x^{1}}^{21}\left(t, x^{1}\right) & \equiv 0,
\end{aligned}
$$

the first bracket in the last term of (41) vanishes as well. Hence, the desired consistency of the above DAE for $x^{3}$ is shown.

In this section we have discussed regularization techniques for various cases of non-regularity. In particular, we have generalized the well-known Filippov regularization to the DAE case.

\section{Examples and numerical experiments}

To illustrate the analysis in the last sections, in this section we present some examples and numerical experiments. We use a C++ implementation of a DAE class for arbitrary index, see [19], using automatic differentiation together with a root finder, extended by an interface for mode switching along the lines described in the previous sections.

Example 16 A two-dimensional pendulum can be modeled via the Hamilton formalism starting with the Hamilton function

$$
H(p, q)=\frac{1}{2} m^{-1} p^{2}-m g L \cos q,
$$

with $m$ the mass, $L$ the length of the pendulum, $g$ the gravity constant, $q$ the generalized position (here the angle between the negative vertical axis and the pendulum), and $p$ the corresponding generalized momentum. In particular, $H$ describes the total energy consisting of kinetic and potential energy. By the Hamilton formalism, the equations of motion are given by

$$
\begin{aligned}
& \dot{p}=-\nabla_{q} H(p, q)=-m g L \sin q, \\
& \dot{q}=\nabla_{p} H(p, q)=m^{-1} p .
\end{aligned}
$$

It is well-known that such a system is conservative, i. e., that the total energy is preserved, due to

$$
\begin{aligned}
\frac{d}{d t} H(p, q) & =\nabla_{p} H(p, q)^{T} \dot{p}+\nabla_{q} H(p, q)^{T} \dot{q} \\
& =-\nabla_{p} H(p, q)^{T} \nabla_{q} H(p, q)+\nabla_{q} H(p, q)^{T} \nabla_{p} H(p, q)=0 .
\end{aligned}
$$

Let $H_{0}$ denote the total energy fixed by the initial values for $p, q$. Then, the switching function

$$
S(t, p, q)=H(p, q)-H_{0}
$$

has the property that the flow connected with the equations of motions lies in the switching surface. Consequently, we are in Case 4.(a) of Section 5. In this example, the only algebraic constraint in the arising overdetermined problem

$$
\dot{p}=-m g L \sin q, \quad \dot{q}=m^{-1} p, \quad H(p, q)-H_{0}=0
$$

has the Jacobian

$$
J=\left[\begin{array}{ll}
m^{-1} p & m g L \sin q
\end{array}\right],
$$

corresponding to $Z_{2}^{T} F_{\mu ; x}$ of Hypothesis 1 . Hence, the variables not fixed by the algebraic constraints are described by

$$
T_{2}=\left[\begin{array}{c}
-m g L \sin q \\
m^{-1} p
\end{array}\right]
$$

again in the notation of Hypothesis 1. Taking $T_{2}$ to project onto the relevant part of the original equations of motion, we end up with the regularized system

$$
\begin{aligned}
-m g L \sin q(\dot{p}+m g L \sin q)+m^{-1} p\left(\dot{q}-m^{-1} p\right) & =0, \\
\frac{1}{2} m^{-1} p^{2}-m g L \cos q-H_{0} & =0 .
\end{aligned}
$$




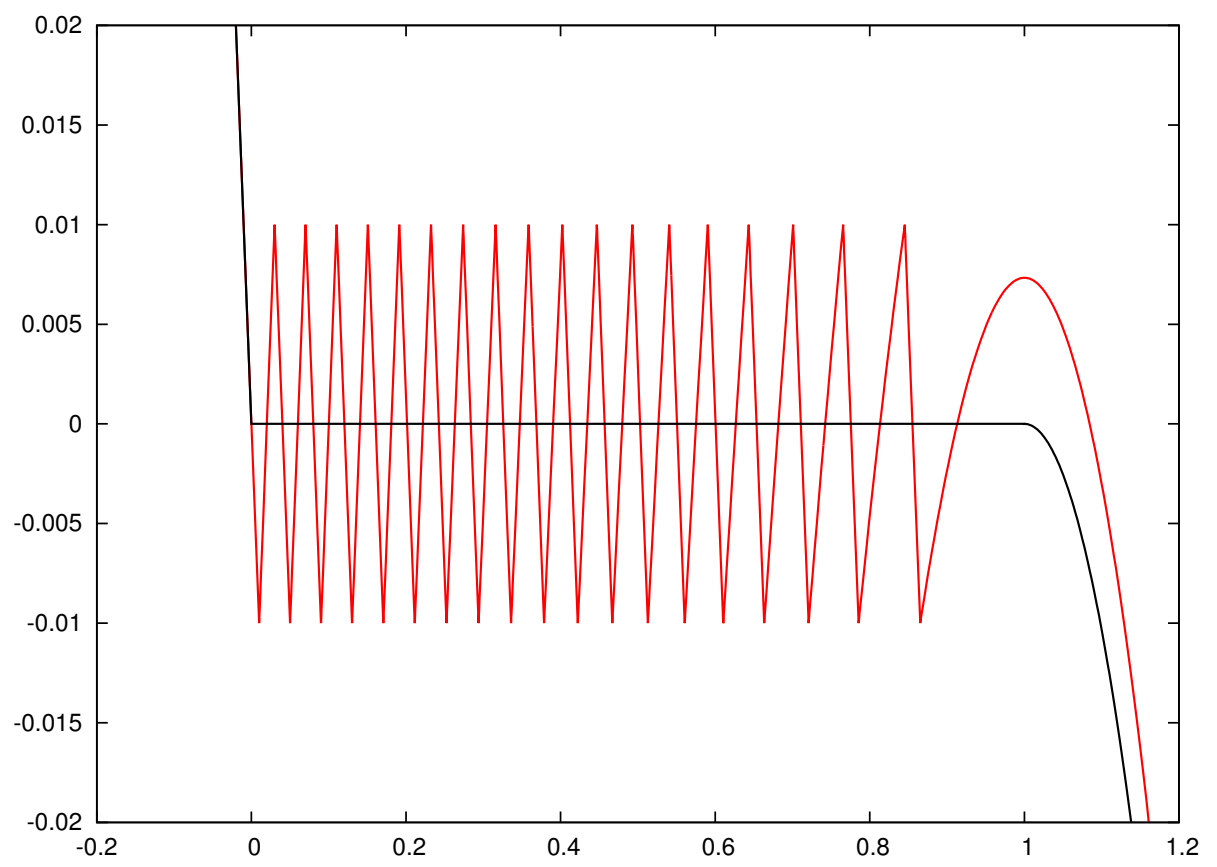

Figure 2: Regularized chattering by generalized Filippov (black) and hysteresis (red)

Example 17 Chattering may occur in the hybrid system

$$
\begin{array}{ll}
\dot{x}_{1}^{1}=-1, \dot{x}_{2}^{1}=0, & \dot{x}_{1}^{2}=1-t^{2} \\
S_{1}^{1}\left(t, x_{1}^{1}, x_{2}^{1}\right)=x_{1}^{1}, & S_{1}^{2}\left(t, x_{1}^{2}\right)=-x_{1}^{2}, \\
T_{1}^{21}\left(t, x_{1}^{1}, x_{2}^{1}\right)=\left(t, x_{1}^{1}, 1-t^{2}\right), & T_{1}^{12}\left(t, x_{1}^{2}\right)=\left(t, x_{1}^{2}, 0,-1,0\right)
\end{array}
$$

for $t \in(-1,1)$. The generalized Filippov regularization as described in the previous section is given by the new mode

$$
\begin{aligned}
& \dot{x}_{2}^{1}=0, \\
& x_{1}^{1}=0, \\
& x_{1}^{1}=x_{1}^{2}, \\
& S_{1}^{3}\left(t, x_{1}^{1}, x_{2}^{1}, x_{1}^{2}\right)=1, \\
& S_{2}^{3}\left(t, x_{1}^{1}, x_{2}^{1}, x_{1}^{2}\right)=1-t^{2}, \\
& T_{1}^{13}\left(t, x_{1}^{1}, x_{2}^{1}, x_{1}^{2}\right)=\left(t, x_{1}^{1}, x_{2}^{1},-1,0\right), \\
& T_{1}^{23}\left(t, x_{1}^{1}, x_{2}^{1}, x_{1}^{2}\right)=\left(t, x_{1}^{2}, 1-t^{2}\right),
\end{aligned}
$$

together with new switching and transfer functions

$$
\begin{array}{ll}
S_{1}^{1}\left(t, x_{1}^{1}, x_{2}^{1}\right)=x_{1}^{1}, & S_{1}^{2}\left(t, x_{1}^{2}\right)=-x_{1}^{2}, \\
T_{1}^{31}\left(t, x_{1}^{1}, x_{2}^{1}\right)=\left(t, x_{1}^{1}, x_{2}^{1}, x_{1}^{1}, 0,0,0\right), & T_{1}^{32}\left(t, x_{1}^{2}\right)=\left(t, x_{1}^{2}, 0, x_{1}^{2}, 0,0,0\right)
\end{array}
$$

for the original modes for $t \in(-1,1)$. Figure 6 shows the solution profile of the first component of all modes for the initial value $\left(t_{0}, z_{0}^{1}\right)=(-1,1,1,-1,0)$ in Mode 1 using the generalized Filippov regularization and hysteresis as described in (26) for $\delta=0.01$.

Example 18 An evaporator vessel can be modeled by the hybrid system

$$
\begin{array}{ll}
I \dot{p}^{1}=-R_{\mathrm{p}} p^{1}, & I \dot{p}^{2}=-R_{\mathrm{p}} p^{2}+L^{2}, \\
C \dot{L}^{1}=-L^{1} / R_{\mathrm{b}}+f_{\mathrm{in}}, & C \dot{L}^{2}=-p^{2} / I-L^{2} / R_{\mathrm{b}}+f_{\mathrm{in}} \\
S^{1}\left(t, p^{1}, L^{1}\right)=L^{1}-L_{\mathrm{th}}, & S^{2}\left(t, p^{2}, L^{2}\right)=-L^{2}+L_{\mathrm{th}}, \\
T^{21}\left(t, p^{1}, L^{1}\right)=\left(t, p^{1}, L^{1}, \dot{p}^{2}, \dot{L}^{2}\right), & T^{12}\left(t, p^{2}, L^{2}\right)=\left(t, p^{2}, L^{2}, \dot{p}^{1}, \dot{L}^{1}\right),
\end{array}
$$




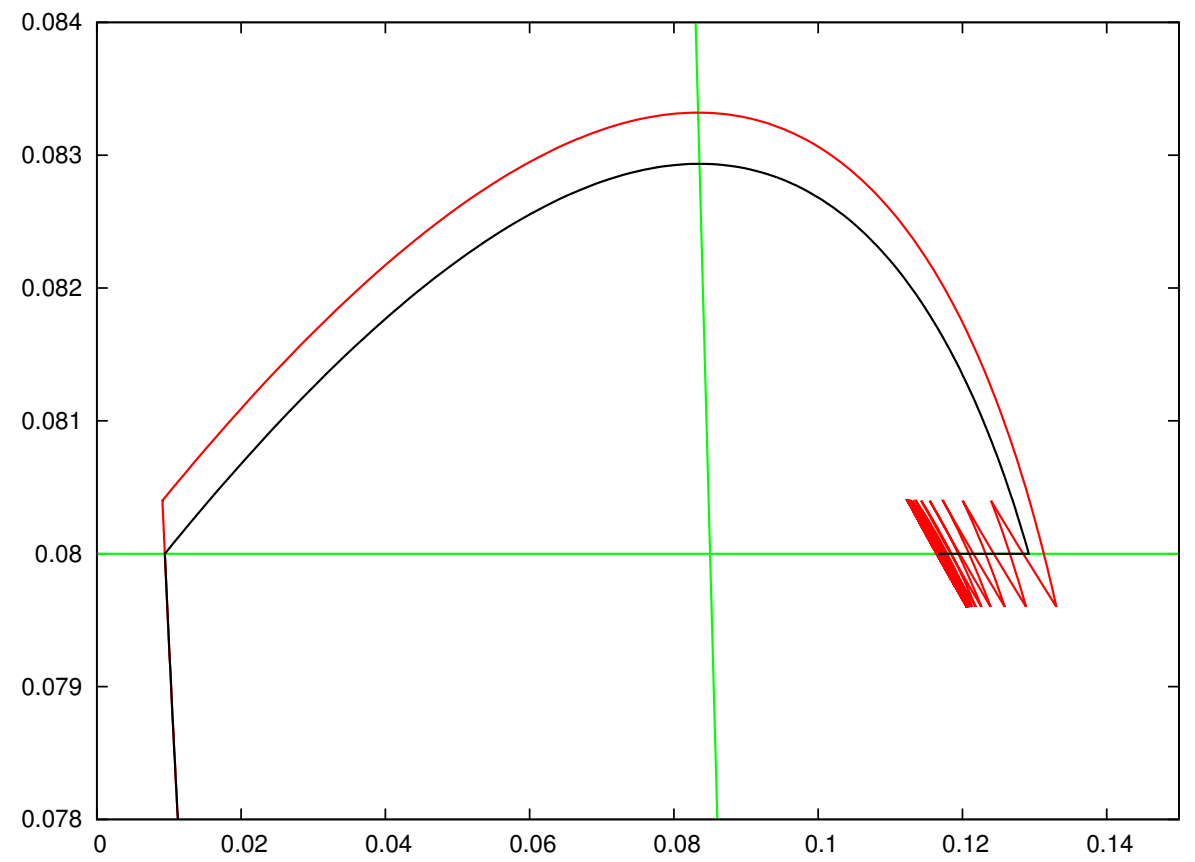

Figure 3: Regularized chattering by generalized Filippov (black) and hysteresis (red)

where the derivatives in the transition functions denote the values obtained by the differential equations of the new mode, see [24] and references therein. The two modes of this example are separated by the line $L=L_{\text {th }}$. With the choice

$$
R_{\mathrm{b}}=1, R_{\mathrm{p}}=0.5, I=0.5, C=15, f_{\mathrm{in}}=0.25, L_{\mathrm{th}}=0.08
$$

of parameters we have that $\dot{L}>0$ in the first mode while in the second mode the line $L=R_{\mathrm{b}}\left(f_{\text {in }}-\right.$ $p / I)$ separates the regions with $\dot{L}>0$ for smaller $p$ and $\dot{L}<0$ for larger $p$. Hence chattering occurs when the solution hits $L=L_{\mathrm{th}}$ in the region to the right of the line $L=R_{\mathrm{b}}\left(f_{\mathrm{in}}-p / I\right)$, cp. Figure 6 . Here, Filippov regularization yields the new mode

$$
\begin{aligned}
& I \dot{p}^{3}=-\alpha R_{\mathrm{p}} p^{3}-(1-\alpha)\left(R_{\mathrm{p}} p^{3}-L^{3}\right), \\
& C \dot{L}^{3}=-\alpha L^{1} / R_{\mathrm{b}}-(1-\alpha)\left(p^{3} / I+L^{3} / R_{\mathrm{b}}\right)+f_{\mathrm{in}}, \\
& L^{3}-L_{\mathrm{th}}=0, \\
& S_{1}^{3}\left(t, p^{3}, L^{3}\right)=p^{3} / I+L^{3} / R_{\mathrm{b}}-f_{\mathrm{in}}, \\
& S_{2}^{3}\left(t, p^{3}, L^{3}\right)=-L^{3} / R_{\mathrm{b}}+f_{\mathrm{in}}, \\
& T^{31}\left(t, p^{3}, L^{3}\right)=\left(t, p^{3}, L^{3}, \dot{p}^{3}, \dot{L}^{3}\right), \\
& T^{32}\left(t, p^{3}, L^{3}\right)=\left(t, p^{3}, L^{3}, \dot{p}^{3}, \dot{L}^{3}\right) .
\end{aligned}
$$

Figure 6 shows the solution in the phase plane $(p, L)$ for the initial value $\left(t_{0}, p_{0}, L_{0}\right)=(0.0,0.05,0.06)$ in Mode 1 using the generalized Filippov regularization and hysteresis as described in (26) for $\delta=0.0004$.

\section{Conclusions}

We have discussed the analysis and numerical solution of hybrid systems of DAEs. It has been shown that it is possible to characterize regular arcs of a solution and how to estimate the stability 
of a global solution. For systems which do not satisfy the regularity assumptions, we have described different regularization techniques, in particular the generalization of Filippov regularization to hybrid systems of DAEs where we even allow that the modes are formulated in different variables. We have illustrated the results via several numerical examples. It is an open problem whether the described cases of non-regularity are a complete set.

\section{References}

[1] J. Agrawal, K.M. Moudgalya, and A.K. Pani. Sliding motion of discontinuous dynamical systems described by semi-implicit index one differential algebraic equations. Chemical Engin. Science, 61:4722-4731, 2006.

[2] J.C. Alexander and T.I. Seidman. Sliding modes in intersecting switching surfaces II: hysteresis. Houston J. Math., 25:185-211, 1999.

[3] Modelica Association. Modelica language specification, version 3.0. Modelica Association.

[4] P.I. Barton and C.K. Lee. Modeling, simulation, sensitivity analysis, and optimization of hybrid systems. ACM Trans. Modeling Computer Simulation, 12:256-289, 2002.

[5] A. Ben-Israel and T. N. E. Greville. Generalized Inverses: Theory and Applications. John Wiley and Sons, New York, NY, 1973.

[6] B. Brogliato. Nonsmooth Mechanics. Springer-Verlag, New York, 1999.

[7] S. L. Campbell. A general form for solvable linear time varying singular systems of differential equations. SIAM J. Math. Anal., 18:1101-1115, 1987.

[8] S. L. Campbell and C. D. Meyer. Generalized Inverses of Linear Transformations. Pitman, San Francisco, CA, 1979.

[9] Dynasim AB, Ideon Research Park - SE-223 70 Lund - Sweden. Dymola,Multi-Engineering Modelling and Simulation, 2006.

[10] E. Eich-Soellner and C. Führer. Numerical Methods in Multibody Systems. Teubner Verlag, Stuttgart, Germany, 1998.

[11] A.F. Filippov. Differential Equations with Discontinuous Right-hand Sides. Kluwer, Dordrecht, 1998.

[12] R. Goebel, R.G. Sanfelice, and A. Teel. Hybrid dynamical systems. Control Systems, IEEE, 29:28-93, 2009 .

[13] E. Hairer, S. P. Nørsett, and G. Wanner. Solving Ordinary Differential Equations I: Nonstiff Problems. Springer-Verlag, Berlin, Germany, 1st edition, 1987.

[14] P. Hamann. Modellierung und Simulation von realen Planetengetrieben. Diplomarbeit, Institut für Mathematik, TU Berlin, Berlin, Germany, 2003.

[15] P. Hamann and V. Mehrmann. Numerical solution of hybrid differential-algebraic equations. Comp. Meth. Appl. Mech. Eng., 197:693-705, 2008.

[16] R. Isermann. Mechatronic systems: concepts and applications. Trans. Institute of Measurement and Control, 22:29-55, 2000.

[17] D. Jeon and M. Tomizuka. Learning hybrid force and position control of robot manipulators. IEEE Trans. Robotic. Automat., 9:423-431, 1996.

[18] T. Krilavicius. Hybrid techniques for hybrid systems. PhD thesis, University of Twente, Enschede, 2006. 
[19] P. Kunkel and V. Mehrmann. Differential-Algebraic Equations. Analysis and Numerical Solution. EMS Publishing House, Zürich, Switzerland, 2006.

[20] C. Lacoursière. Ghosts and Machines: Regularized Variational Methods for Interactive Simulation of Multibodies with Dry Frictional Contacts. Phd thesis, Umeå University, Sweden, 2007.

[21] S. Lang. Analysis I. Addison-Wesley, Reading, MA, 3rd edition, 1973.

[22] J. Lunze and F. Lamnabhi-Laguarrigue. Handbook of Hybrid Systems Control. Theory, Tools and Applications. Cambridge University Press, Cambridge, UK, 2009.

[23] J. Lygeros, G.J. Pappas, and S. Sastry. An Introduction to Hybrid System Modeling, Analysis, and Control. Preprints of the First Nonlinear Control Network Pedagogical School, Athens, Greece, pages 307-329, 1999.

[24] V. Mehrmann and L. Wunderlich. Hybrid systems of differential-algebraic equations - analysis and numerical solution. J. Process Control, 19:1218-1228, 2009.

[25] L. Wunderlich. Analysis and Numerical Solution of Structured and Switched DifferentialAlgebraic Systems. Phd thesis, TU Berlin, 2008.

[26] M. Yu, L. Wang, T. Chu, and G. Xie. Stabilization of networked control systems with data packet dropout and network delays via switching system approach. In IEEE Conference on Decision and Control, pages 3539-3544, 2004. 